\title{
Designing Tasks of Introductory Real Analysis to Bridge a Gap Between Students' Intuition and Mathematical Rigor: the Case of the Convergence of a Sequence
}

\author{
Kyeong Hah Roh $^{1}$ • Yong Hah Lee ${ }^{2}$
}

Published online: 28 October 2016

(C) Springer International Publishing Switzerland 2016

\begin{abstract}
The purpose of this study is to explore how an introductory real analysis (IRA) course can be designed to bridge a gap between students' intuition and mathematical rigor. In particular, we focus on a task, called the $\varepsilon$-strip activity, designed for the convergence of a sequence. Data were collected from a larger study conducted as a classroom teaching experiment for a semester-long IRA course. Fischbein's notion of secondary intuition was employed to elucidate the development of student intuition throughout the $\varepsilon$-strip activity. We discuss how the $\varepsilon$-strip activity played a role in developing students' intuition and how it impacted students' learning of the subsequent topics in the course, including definitions and theorems about convergence.
\end{abstract}

Keywords Convergence of a sequence - Introductory real analysis · Intuition · Definition · Proving · Task design

\section{Introduction}

Introductory real analysis (IRA), or advanced calculus, is mandatory for most university students majoring in mathematical sciences (e.g., Pollatsek et al. 2004). Through the course, undergraduate students are expected to understand the theoretical

Kyeong Hah Roh

khroh@asu.edu

Yong Hah Lee

yonghah@ewha.ac.kr

1 School of Mathematical and Statistical Sciences, Arizona State University, P.O. Box 871804, Tempe, AZ 85287-1804, USA

2 Department of Mathematics Education, Ewha Womans University, Seoul 120-750, South Korea 
foundation of calculus conceptually and to construct arguments and rigorous proofs of calculus theorems. In particular, the $\varepsilon-N$ definition ${ }^{1}$ of convergent sequences is fundamental in studying IRA. Undergraduate students' conceptualization of the $\varepsilon-N$ definition has been studied by a number of researchers for the past decade (e.g., Cory and Garofalo 2011; Dawkins 2012; Oehrtman et al. 2014; Pinto and Tall 2002). Many students experience difficulty in understanding and applying the $\varepsilon-N$ definition to prove theorems in IRA (Dawkins and Roh 2016; Roh and Lee 2011). Research indicates that students' difficulties with learning the $\varepsilon-N$ definition are related to the incompatibility between their intuitive understanding of convergence and such a rigorous definition (e.g., Cornu 1991; Davis and Vinner 1986; Roh 2008a, 2010).

The purpose of this study is to explore how an IRA course could bridge a gap between students' intuitive understanding and mathematical rigor. In particular, we focus on an activity, called the $\varepsilon$-strip activity, designed to teach the $\varepsilon-N$ definition of convergence in an inquiry-oriented IRA class. Three classroom episodes are reported in this paper to address the following research questions:

(1) Through the $\varepsilon$-strip activity, how do students develop a new intuition, called secondary intuition by Fischbein (1987), about convergence?

(2) How does students' secondary intuition, developed through the $\varepsilon$-strip activity, play a role in understanding the $\varepsilon-N$ definition?

(3) How does students' secondary intuition, developed through the $\varepsilon$-strip activity, play a role in proving a theorem related to convergence?

\section{Teaching and Learning of the Convergence of a Sequence in the IRA Context}

The convergence of a sequence is one of the foundational concepts in IRA (Alcock 2010; Alcock and Weber 2005; Barne 2007; Jorgensen and Shipman 2012; Shipman 2012; Weber 2004). In particular, the $\varepsilon-N$ definition is dealt with in IRA courses to rigorously define the convergence of a sequence in terms of a specific relationship between $\varepsilon$ and $N$. Comprehension of the relationship between $\varepsilon$ and $N$ plays a crucial role in properly understanding the convergence of a sequence (Cory and Garofalo 2011; Dawkins 2012; Oehrtman et al. 2014; Mamona-Downs 2001; Roh 2010; Roh and Lee 2011). On the other hand, it has been known as a challenge for many students to grasp the relationship between $\varepsilon$ and $N$ from the $\varepsilon-N$ definition (e.g., Davis and Vinner 1986; Edwards 1997; Knapp 2006). In particular, students tend to regard $\varepsilon$ and $N$ as unnecessary in defining the limit of a sequence (Cory and Garofalo 2011; Mamona-Downs 2001), or incorrectly conceptualize the relationship between them (Dawkins 2012; Pinto and Tall 2002; Roh 2010; Roh and Lee 2011). For instance, some do not construe that the value of $N$ can be selected depending on the value of $\varepsilon$. These students do not cognize that the value of $N$ can vary accordingly as $\varepsilon$ varies (Durand-Guerrier and Arsak 2005). On the other hand, among even those who construe the dependence of $N$ on $\varepsilon$, some students tend to

\footnotetext{
${ }^{1}$ In this paper we refer to the following statement as the $\varepsilon-N$ definition: A sequence $\left\{a_{n}\right\}_{n=1}^{\infty}$ converges to $L \in \mathbf{R}$ if for any $\varepsilon>0$, there exists $N \in \mathbf{N}$ such that for all $n>N,\left|a_{n}-L\right|<\varepsilon$.
} 
think that the value of $\varepsilon$ can also be determined based on the value of $N$, just as $N$ can be determined based on $\varepsilon$ (Roh and Lee 2011). In addition, some students do not infer the arbitrariness of error bounds from the $\varepsilon-N$ definition. They tend to test with only some $\varepsilon>0$ for the condition "for all $n>N,\left|a_{n}-L\right|<\varepsilon$ " instead of considering every $\varepsilon>0$ (Dawkins 2012; Oehrtman et al. 2014; Roh 2010). Students do not often grasp that "any $\varepsilon$ " implies the value of $\varepsilon$ decreases towards 0 so that smaller values can be continuously chosen for $\varepsilon$ (Roh 2010).

Indeed, the relationship between $\varepsilon$ and $N$ is not explicitly stated in the $\varepsilon-N$ definition. For instance, $N$ does not appear in the inequality " $\left|a_{n}-L\right|<\varepsilon$ " in the $\varepsilon-N$ definition. Furthermore, an explicit algorithm to find the value of $N$ is not suggested in the $\varepsilon-N$ definition. As a consequence, it might not be easy for students to reason properly why $\varepsilon$ and $N$ are necessary in defining the convergence of a sequence, how $N$ could be determined dependently on $\varepsilon$, and why $\varepsilon$ must be independent of $N$ (Cory and Garofalo 2011; Dawkins 2012; Mamona-Downs 2001; Roh and Lee 2011).

In order to improve students' capacity to conceptualize the relationship between $\varepsilon$ and $N$ in the $\varepsilon-N$ definition, mathematics educators have stressed the role of visualization (Cheng and Leung 2015; Cory and Garofalo 2011; Mamona-Downs 2001; Navarro and Carreras 2006; Pinto and Tall 2002; Roh 2008a). They further proposed that delivering conceptual ideas inherent in the $\varepsilon-N$ definition with graphical illustrations would help students understand the unstated semantics from the definition. For example, Mamona-Downs (2001) suggested rephrasing the $\varepsilon-N$ definition as follows: $L$ is the limit of a sequence if "given $\varepsilon$, there is a certain position beyond which all further terms of the sequence are within the distance $\varepsilon$ from $L$ " (p. 278). As another way to visualize the $\varepsilon-N$ definition, Roh (2008b) introduced an " $\varepsilon$-strip" as a physical model of the region bounded by two horizontal lines with distance $\varepsilon$ from the line $y=L$. Using the physical device, the $\varepsilon-N$ definition was rephrased as follows: $L$ is the limit of a sequence if "for any $\varepsilon$-strip, only finitely many terms of the sequence are outside the $\varepsilon$-strip when the $\varepsilon$-strip is centered at $L$ " (p.81). Other studies also advocated the advantage of rephrasing the $\varepsilon-N$ definition with a visual representation (e.g., Cory and Garofalo 2011; Dawkins 2012) similar to $\varepsilon$-strips. In line with this perspective, this study implemented Roh's (2008b) $\varepsilon$-strip activity to an IRA classroom designed for an inquiry-based learning. In this paper, we address how the $\varepsilon$-strip activity could help students develop their intuition to be compatible with the $\varepsilon-N$ definition and how it would impact their learning of subsequent topics about convergence throughout the course.

\section{Theoretical Perspective}

The IRA course reported in the paper was developed based on a theoretical standpoint toward mathematical intuition as cognitive capacity. One might overlook the importance of mathematical intuition in the IRA course because the mathematics dealt in the course is assumed to be a formal and rigorous body of knowledge. We agree that formalization or rigor is essential in representing the logically structured mathematical knowledge in the IRA course. However, pursuing precision and formalization tend to be applied mostly to the final phase of mathematical activities. Many talented mathematicians actively utilized their mathematical or scientific intuition about the essence of an idea before rigorously justifying or formalizing the idea (Burton 1999; Hadamard 
1996). Their intuition guided to unveil the essence of mathematical knowledge (Fischbein 1987, 1994, 1999). The utilization of intuitive ideas occurs universally in many mathematical fields. In addition, intuitive ideas often open a new area in mathematics and provide a clue to the development of a mathematical topic (Fischbein 1987; Hadamard 1996). We therefore presume that there is a different kind of mental activities other than formal cognitive processes operating in mathematical activities that IRA students also need to develop.

To put it concretely, the present study calls it as intuitive cognition or intuition, referring to cognition directly accepted without or prior to any rigorous justification. In addition, the term intuitive understanding of a concept in this paper simply means immediately cognizing the concept without any kind of rigorous processes. Our definition of intuition is aligned with Fischbein's (1994) definition as the "immediate cognition that is accepted directly without the feeling that any kind of justification is required" (p. 232). Indeed, Fischbein (1987) characterizes intuitions as self-evident and intrinsically certain. Such characteristics of intuition often entail an individual's immediate response or direct acceptance to a problem without or prior to any rigorous justification. In line with the standpoint, in this study we consider students' immediate perception without rigorous justification as possible evidence of them applying their intuitive ideas to the problem situation.

\section{Secondary Intuition About the Convergence of a Sequence}

Fischbein (1987) classifies intuition into primary intuition and secondary intuition via its origin. Primary intuition refers to intuitive cognition that "develops in individuals independently of any systematic instruction as an effect of their personal experience" (p. 64). All representations and interpretations developed prior to systematic instruction of a concept can be considered as products of primary intuitive cognition of the concept. In the case of IRA context, we consider students' primary intuition as intuition that has already developed independently of IRA instruction. For instance, students' intuitive understanding of convergence before the $\varepsilon-N$ definition being taught is considered their primary intuition. It does not mean that these students had never received any instruction for convergence before they receive a systematic instruction for the $\varepsilon-N$ definition. In fact, many students in IRA would have already taken calculus as a prerequisite for the IRA course and they would have received a systematic instruction for the convergence from the calculus courses. For instance, calculus students would have acquainted the limit of a sequence $\left\{a_{n}\right\}$ of real numbers as a real number that the sequence $a_{n}$ approaches as $n$ increases towards infinity (e.g., Stewart 2003). However, if the $\varepsilon-N$ definition was not exposed to the students in their calculus courses, we consider that the students develop their intuition of convergence independently of any systematic instruction for the $\varepsilon-N$ definition; hence, in that case, we consider their intuition of convergence as their primary intuition.

On the other hand, once students learn the $\varepsilon-N$ definition through systematic instruction and it becomes self-evident to the students, we then consider that the students acquire secondary intuition. For students who completed calculus but did not take IRA course, the $\varepsilon-N$ definition might be considered as incompatible with their intuitive understanding of convergent sequences (Roh 2008a). Hence, these students consider the $\varepsilon-N$ definition as very strange or counter-intuitive (Courant and Robbins 
1963; Mamona-Downs 2001). However, if appropriate tasks and instructional interventions are given, students' secondary intuition about convergence could be developed to be compatible with the $\varepsilon-N$ definition. By obtaining such a secondary intuition, the students would no longer feel that the $\varepsilon-N$ definition counters their intuition. In this classification of intuition, the point to be aware of is that secondary intuition can be obtained and developed (Bruner 1960; Fischbein 1987). We even further expect secondary intuition could be developed to be compatible with mathematical rigor.

\section{Students' Development of Secondary Intuition in IRA Classrooms}

One of the merits of intuitive understanding is that it gives a person self-confidence, conviction, or certainty toward his or her reasoning. Fischbein (1987) insists that when one fails to intuitively grasp a concept, he or she would be unsure of own reasoning even after solving a problem about the concept. Szydlik's (2000) study also indicates that students who intuitively understood the concept of limit tended to trust their decision in solving problems. We additionally posit that the compatibility between students' intuition and mathematical rigor would reinforce the students' conviction toward their reasoning. The IRA course reported in this paper was thus designed to provide appropriate activities to students so that their secondary intuition could be developed to be compatible with mathematical rigor. In this paper, we focus on how students' secondary intuition about convergence could be built up while engaged in the activities of IRA course.

In fact, intuition is considered as it belongs to a personal cognitive process in the sense that individuals would form their own personal intuition. Fischbein (1987) even contends that primary intuition for someone may actually be secondary intuition for others. However, through continued communication in a learning community, individual students' understanding could contribute to build upon the learning community's collective understanding (Martin et al. 2006; Rasmussen and Stephan 2008; Vidakovic and Martin 2004). This is what Tabach et al. (2014) called collective activities of a classroom community, or "normative ways of reasoning that are developed in a classroom community" (p. 195). We often observed the frequent occurrence of such phenomena in the IRA classes structured for students to work in groups. To be more precise, there were moments in the classes that a group of students immediately accepted an idea without any rigorous justification. This idea tended to function as taken-as-shared knowledge (Yackel and Cobb 1996) or it functioned as if it is shared knowledge by the group of students (Rasmussen and Stephan 2008), even though individual variations still existed within the group. Hence, students' intuitive understanding illustrated in this paper refers to collective intuitive knowledge constructed and accepted by students in a group.

\section{Research Methodology}

\section{Classroom Teaching Experiments}

This study was conducted from 2006 to 2010 at a large university in the United States as design research (Cobb et al. 2001) involving three iterations of instructional design 
and exploratory teaching experiments (Steffe and Thompson 2000) in IRA classrooms. We chose this research methodology primarily to explore students' mathematical learning and reasoning in IRA classrooms. By the exploratory nature of the teaching experiments, we attempt to model how students' conceptions and reasoning have evolved as they engaged in IRA class activities. The iterative nature of the teaching experiments allowed frequent research cycles as follows: (a) Addressing issues or problems in the learning of specific topics in IRA, (b) designing/refining instructional materials, (c) predicting student learning, (d) implementing the designed tasks in class, and (e) analyzing students' actual learning through the tasks. Hence, this research methodology allowed us to design instructional materials to support student learning, to systematically study the processes of refining and developing the instructional products, and to demonstrate the results of student learning in IRA.

Data consisted of videotape recordings and copies of students' written work, some of which were synchronized with the students' voices during group discussion, videotape recordings of task-based interviews with individual students, and copies of individual students' weekly homework and exams. During class sessions there were 2-3 cameras, each focused on a different group of students. Table 1 summarizes the three iterations of the classroom teaching experiments.

In this paper, we focus on the final version of the instructional sequences implemented to Cohort 3 in spring 2010. The first author of the present paper served as the course instructor and eleven students participated in the cohort, forming three small groups (four students in Groups 1 and 2, and three students in Group 3). This cohort consisted of both high- and low-performing students from prerequisite mathematics courses.

Prior to participating in this study, the students already completed calculus for real functions and introductory proof courses. However, they had received no formal instruction for IRA topics such as the $\varepsilon-N$ definition of the convergence of a sequence. Hence, we assumed the participants had primary intuition but had not yet developed secondary intuitive understanding of topics in IRA.

In the IRA course a conventional lecture style instruction was minimized to create an environment for student-centered inquiry-based learning (IBL). To facilitate student inquiries, students were often expected to contribute in knowledge construction. For instance, the $\varepsilon-N$ definition was not introduced to the class until students explored the meaning of the convergence of a sequence throughout group discussion. After the topics were explored in group, more detailed class-notes (e.g., a list of definitions and theorems) were handed out to students and the instructor led class discussion so as for students to attend to other groups' ideas and to resolve conflicts between various ideas if necessary.

Table 1 Summary of the exploratory teaching experiments

\begin{tabular}{lllll}
\hline Cohort & $\begin{array}{l}\text { Semester } \\
(15 \text { weeks })\end{array}$ & Sessions per week & $\begin{array}{l}\text { \# of students } \\
\text { (videotaped) }\end{array}$ & $\begin{array}{c}\text { \# of groups } \\
\text { (videotaped) }\end{array}$ \\
\hline 1 & Fall 2006 & Three 50-min classes & $6(6)$ & $2(2)$ \\
2 & Spring 2007 & Two 75-min classes & $20(12)$ & $5(3)$ \\
3 & Spring 2010 & $\begin{array}{l}\text { Two 75-min classes and one 50-min } \\
\text { session for collaborative proof writing }\end{array}$ & $11(11)$ & $3(3)$ \\
\hline
\end{tabular}




\section{A Case of the Convergence of a Sequence: the $\varepsilon$-strip Activity}

One of the tasks that we implemented in the IRA course was "the $\varepsilon$-strip activity" (Roh $2008 b)$ related to the $\varepsilon-N$ definition. The $\varepsilon$-strips were introduced to students as strips with constant width, $2 \varepsilon$, and indefinite length. The physical model of the $\varepsilon$-strips was made of translucent paper so as to observe the graph of a sequence through the $\varepsilon$-strips. In addition, a red line was drawn in the center of each $\varepsilon$-strip so as to mark a value to be tested for the limit of a sequence.

During the $\varepsilon$-strip activity, students were first asked to determine if given sequences in Table 2 have limits and then to state their criteria in determining the convergence. These sequences were selected as examples of sequences to be (i) monotone convergent, (ii) constant after finite terms, (iii) oscillating convergent with a constant subsequence, (iv) oscillating divergent with an unbounded subsequence and a convergent subsequence, $(v)$ oscillating divergent with a constant subsequence, ( $v i)$ oscillating convergent, and (vii) oscillating divergent with two cluster points (See Table $2^{2}$ ). At this point, we expected for students to use their primary intuition about convergence as they had not yet acquainted with convergence via the rigorous definition.

The $\varepsilon$-strips were introduced to students after they realized that their primary intuition was problematic (e.g., a conception of convergence as 'approaching but not reaching' was not adequate) and they agreed with the necessity of a better criterion for determining the convergence of a sequence. The main step of the $\varepsilon$-strip activity was to evaluate $\varepsilon$-strip definitions A and B as hypothetical students' conjectures:

$\varepsilon$-strip definition A: $L$ is a limit of a sequence when for any $\varepsilon$-strip, infinitely many points on the graph of the sequence are inside the $\varepsilon$-strip as long as the $\varepsilon$-strip is centered at $L$.

$\varepsilon$-strip definition B: $L$ is a limit of a sequence when for any $\varepsilon$-strip, only finitely many points on the graph of the sequence are outside the $\varepsilon$-strip as long as the $\varepsilon$ strip is centered at $L$.

The process of counting the number of terms of a sequence outside a given $\varepsilon$-strip, called the counting process in Roh's (2010) study, plays an important role in students' understanding of $\varepsilon$-strip definitions $\mathrm{A}$ and $\mathrm{B}$. In addition, the counting process is equivalent to the process of determining a value of $N$ for a given error bound $\varepsilon$ in the $\varepsilon-N$ definition. Thus, before evaluating $\varepsilon$-strip definitions $\mathrm{A}$ and $\mathrm{B}$, students were asked to manipulate various $\varepsilon$-strips of different widths on the same graph of a sequence to cover a possible limit value. They were also asked to determine how many points on the graph of the sequence are outside and then inside each $\varepsilon$-strip.

After providing enough opportunities to work with $\varepsilon$-strips and the graphs of the sequences in Table 2, we introduced two hypothetical students' arguments, each of which falsely determines that the sequence $\left\{\frac{1}{n}\right\}$ converges to -0.05 as follows:

Ben: Choose an $\varepsilon$-strip with $\varepsilon=0.1$ and align its center at $y=-0.05$ on the graph of the sequence $\left\{\frac{1}{n}\right\}$. Then infinitely many points on the graph of the sequence are

\footnotetext{
${ }^{2}$ Similar sequences to those in Table 2 were found from other studies on student understanding of convergent sequences or the limit of a sequence (Cory and Garofalo 2011; Przenioslo 2004; Oehrtman et al. 2014).
} 
Table 2 Sequences provided to students during the $\varepsilon$-strip activity

\begin{tabular}{|c|c|c|}
\hline (i) $a_{n}=1 / n$ & (ii) $a_{n}=\left\{\begin{array}{l}1 / n \text { if } n \leq 10 \\
1 / 10 \text { if } n>10\end{array}\right.$ & (iii) $a_{n}= \begin{cases}1 / n & \text { if } n \text { is odd; } \\
0 & \text { if } n \text { is even. }\end{cases}$ \\
\hline (iv) $a_{n}= \begin{cases}1 / n & \text { if } n \text { is odd } \\
n & \text { if } n \text { is even }\end{cases}$ & (v) $a_{n}= \begin{cases}1 / n & \text { if } n \text { is odd; } \\
1 & \text { if } n \text { is even. }\end{cases}$ & $\begin{array}{l}\text { (vi) } a_{n}=(-1)^{n} / n \\
\text { (vii) } a_{n}=(-1)^{n}(1+1 / n)\end{array}$ \\
\hline
\end{tabular}

inside the $\varepsilon$-strip. Hence, accepting the statement $\mathrm{A}$ as a definition of limits, we should determine the value -0.05 as a limit of the sequence $\left\{\frac{1}{n}\right\}$.

Emma: Choose an $\varepsilon$-strip with $\varepsilon=0.1$ and align its center at $y=-0.05$ on the graph of the sequence $\left\{\frac{1}{n}\right\}$. Then only finitely many points on the graph of the sequence are outside the $\varepsilon$-strip. Hence, accepting the statement B as a definition of limits, we should determine the value -0.05 as a limit of the sequence $\left\{\frac{1}{n}\right\}$.

These two hypothetical students' arguments above include the same logical flaw that only one $\varepsilon$-strip with $\varepsilon=0.1$ was examined when applying $\varepsilon$-strip definitions $\mathrm{A}$ and $\mathrm{B}$, respectively (Fig. 1). In fact, if a narrower $\varepsilon$-strip with $\varepsilon \leq$ 0.05 is aligned at $y=-0.05$, a finite number of points are inside the $\varepsilon$-strip and infinitely many points are outside the $\varepsilon$-strip (Fig. 2); thus unlike Ben's and Emma's arguments, accepting $\varepsilon$-strip definitions $\mathrm{A}$ and $\mathrm{B}$ does not determine the value -0.05 as a limit of the sequence $\left\{\frac{1}{n}\right\}$. Roh's (2010) study indicated that, similar to Ben and Emma, calculus students tended to interpret the meaning of the word "any $\varepsilon$-strip" in $\varepsilon$-strip definitions as "some $\varepsilon$-strip." These students did not conceive of the arbitrary nature of $\varepsilon$-strips in $\varepsilon$-strip definitions. As a consequence of such a non-conventional interpretation of the words 'any $\varepsilon$-strip' in $\varepsilon$-strip definitions $\mathrm{A}$ and $\mathrm{B}$, some students would inappropriately evaluate $\varepsilon$-strip definitions. Hence, students needed guidance to conceptualize the arbitrariness of the $\varepsilon$ strips in $\varepsilon$-strip definitions prior to evaluating $\varepsilon$-strip definitions $\mathrm{A}$ and B. For this purpose, we introduced Ben and Emma's arguments to the class and asked students to evaluate these arguments. Indeed, group discussion for the evaluation of these invalid arguments provided a chance for students to mentally visualize all possible widths of $\varepsilon$-strips from their physical operation of only a few $\varepsilon$-strips. In addition, for those who accounted only for some $\varepsilon$-strips, group discussion also served as a catalyst to conceptualize the arbitrariness of $\varepsilon$-strips by listening to other students' interpretation of the meaning of 'any $\varepsilon$-strip' in $\varepsilon$-strip definitions $\mathrm{A}$ and $\mathrm{B}$.

Finally, students were then asked to evaluate $\varepsilon$-strip definitions A and B. Students were also asked to construct a new statement or to revise the $\varepsilon$-strip definitions if they thought these statements are not good enough as definitions of a convergent sequence. While students worked in small groups for this activity, the instructor visited them to monitor their progress and provided minimal, but necessary, instructional prompts.

\section{Tasks After the $\varepsilon$-strip Activity}

After the $\varepsilon$-strip activity, the instructor led the whole class discussion to collect and share each small group's work. The rigorous $\varepsilon-N$ definition was introduced after the 


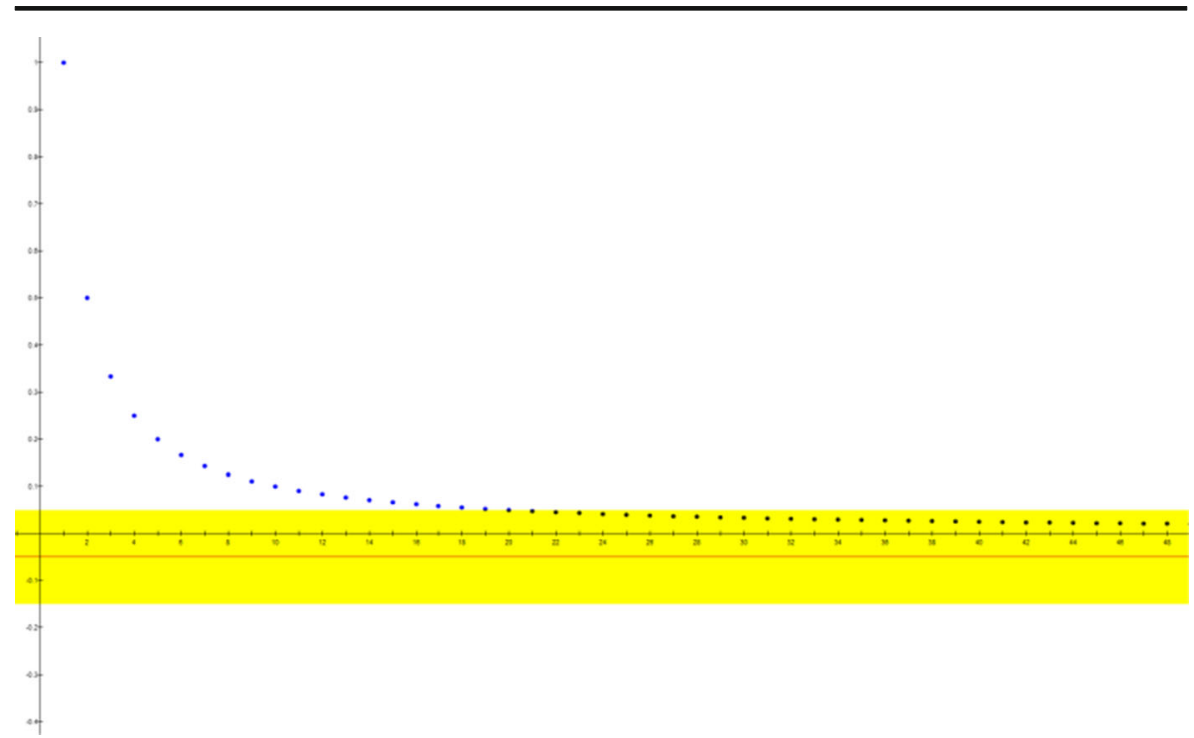

Fig. 1 Only finitely many terms of $\{1 / n\}$ are outside an $\varepsilon$-strip centered at $y=-0.05$

class accepted $\varepsilon$-strip definition $\mathrm{B}$ as a definition of the convergence of a sequence. For a few weeks from that day, the class work centered on proving statements about convergent sequences. Those statements were about the convergence of specific sequences in Table 2, and relationships among convergent sequences, bounded sequences, monotone sequences, and Cauchy sequences. The students were often requested by the instructor to interpret their proofs by using not only the $\varepsilon-N$ definition but also $\varepsilon$-strip definition B.

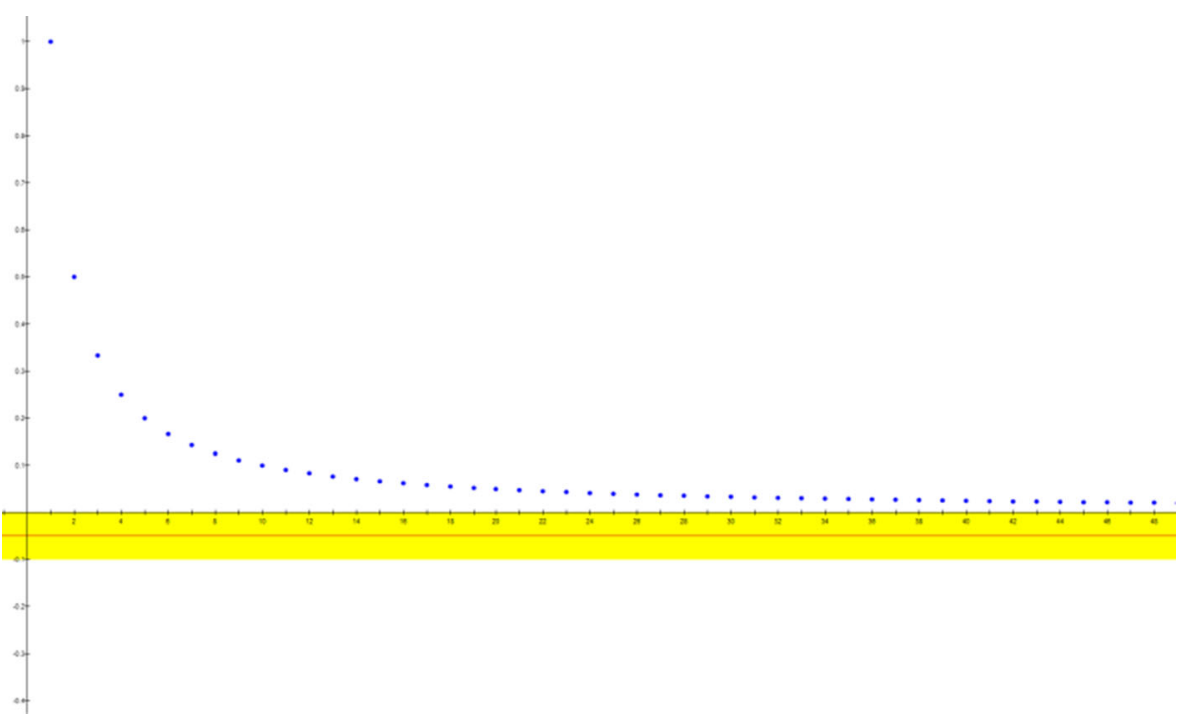

Fig. 2 Infinitely many terms of $\{1 / n\}$ are outside an $\varepsilon$-strip centered at $y=-0.05$ 


\section{Description of Data Analysis}

Our data analysis was conducted in the spirit of grounded theory (Strass and Corbin 1998). Hence, the categories of student ideas and reasoning reported in this paper were not from the researchers' a priori analysis but they emerged from the classroom data.

The first step of the data analysis involved reviewing the classroom data, i.e., videorecordings of both group discussions and whole-class discussions, transcripts of these video-recordings, and each group's written work, synchronized with voices of the students in the group. As an independent coder, each author of the present paper went through an open-coding process to identify students' ideas and reasoning. In particular, each of us paid attention to identify when a new idea or claim emerged (time stamp), what the idea/claim was (claim), which student spoke about the idea/claim first in which group (student name and group number), and how the student explained the idea/claim (e.g., types of sequences).

The second step of the data analysis involved refining the categories of student ideas, claims, and reasoning. For the purpose of viability of our categorization, we compared two sets of coding created independently by each author (coder), and discussed whenever disagreement in coding was found between the coders. This discussion continued until a consensus was reached for each category of student ideas and claims. For instance, by the end of the second step of the analysis for the $\varepsilon$-strip activity, we categorized students' evaluation of $\varepsilon$-strip definitions in terms of (1) the correctness of each $\varepsilon$-strip definition as a definition of convergent sequences; (2) the sufficiency of Conditions A and B; and (3) the necessity of Conditions A and B in defining the convergence of a sequence, where Conditions $\mathrm{A}$ and $\mathrm{B}$ are stated as follows:

- Condition A: For any $\varepsilon$-strip, infinitely many points are inside the $\varepsilon$-strip.

- Condition B: For any $\varepsilon$-strip, only finitely many points are outside the $\varepsilon$-strip.

In this paper, we will simply use words "correct", "sufficient", and "necessary" so long as the meanings are clear in the context. That is, when we say in the analysis "students evaluated the statement is correct," we mean "students evaluated (said) the statement is correct as a definition of convergent sequences." Similarly, when we describe students' evaluation of conditions as "necessary" or "sufficient," we mean "necessary, or sufficient, in defining the convergence of a sequence." Note that we used these codes not with their logical meanings but based on the students' utterance. For instance, students often said a condition is necessary in defining the convergence, which might mean for the students that the condition needs to appear to a definition of convergence. We then coded that the students evaluated the condition as "necessary."

At the third step of the data analysis, we looked at the codes across various points within each class session and across class sessions. The main purpose of the analysis at this step was to find any changes in students' ideas, claims, and reasoning. We first conducted this analysis for each group, and later compared similarities and differences among the groups of the class. Table 3 is an example of the analysis at this phase for the $\mathcal{E}$-strip activity with Cohort 3 in Spring 2010. 
Table 3 Summary of the evolution of student evaluations of $\varepsilon$-strip definitions A and B (Cohort 3, Spring 2010)

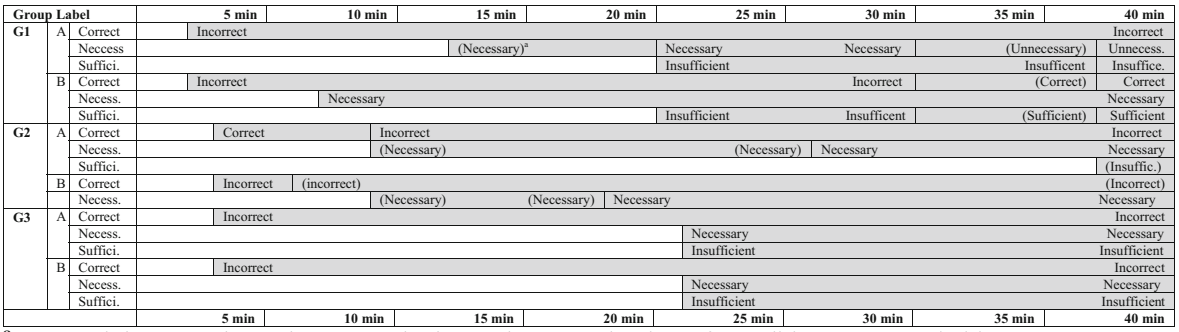

a We used the parentheses here to code the students' evaluation of Condition A as "probably" necessary. In a similar manner, we use the parentheses in this table.

Finally, we coded students' claims or ideas as intuitive understandings if they were immediately accepted without any rigorous justification by at least one student of a group. In addition, we considered students' hand gestures, diagrams, and wordings used to describe an image as observable behaviors of the students' intuitive cognition whereas analytic or algebraic manipulations were considered as their formal cognitive activities. For instance, when interpreting the $\varepsilon-N$ definition, a student drew a pair of horizontal lines and said "all of these [lines] get closer and closer" while contracting her right hand to form a fist. We considered her hand motions, drawing of two horizontal lines, and her word expressions are all indicative of her imagination of the width of $\varepsilon$-strips decreasing towards 0 . A student's gestures, diagrams, and wordings are not rigorous or formal cognitive activities; however, these behaviors serve the function of conveying the student's intuitive understanding of the $\varepsilon-N$ definition. Furthermore, we view that the student's gestures, diagrams, and verbal descriptions play an important role in helping her communicate with other students in her group (e.g., Rasmussen et al. 2004; Raynolds and Reeve 2002).

If a student's intuitive understanding was accepted by all members of a group, we identified the idea as the group's collective intuitive understanding. In particular, we adopted Rasmussen and Stephan's (2008) two criteria for a normative way of reasoning: A mathematical idea expressed in an argument is considered as self-evident when none of the group members is challenged by the lack of analytical explanations or rigorous justification of the idea; and the mathematical idea is considered as functioning as if it were shared when students use a previously justified claim as unchallenged justification for future arguments. The first criteria, self-evidence, was used to determine the intuitive nature; and the second criteria, functioning as if shared, was used to determine the collective nature.

\section{Results: the Case of Group 1}

In this paper, we report three classroom episodes to provide insight into the development of students' intuitive understanding and its role in learning definitions and theorems in an IRA course. Episode 1 is from Day 8 when the $\varepsilon$-strip activity was implemented in the IRA class. In line with the first research question, this episode was 
chosen to illustrate the development of students' secondary intuition about the convergence of sequences via the $\varepsilon$-strip activity. Episode 2 is from Day 9, right after the $\varepsilon$-strip activity was implemented. In line with the second research question, this episode was chosen to demonstrate the role of students' secondary intuition in conceptualizing the $\varepsilon-N$ definition. Episode 3 is from Day 14, 2 weeks from the day of Episode 2. In line with the third research question, this episode illustrates the role of students' secondary intuition in proving a statement about convergent sequences. Prior to the three classroom episodes, we also reported the instructional sequence and students' work in Day 7, which was the day before the $\varepsilon$-strip activity was implemented in the IRA class. This description of Day 7 provides students' primary intuition about the convergence of a sequence as a basis for the development of the students' intuitive understanding during the subsequent three classroom episodes.

Each classroom episode in this section was organized in terms of phases. These phases were made through our data analysis as we found any changes or shift in the students' ideas and thinking. Hence, whereas the classroom episodes were determined by specific topics and curricular activities, the phases were determined based on students' responses to these curricular activities. Hence, the number of phases and the description of each phase would be different for a different group of students for the same classroom episode.

In this paper, we focus on a group of students, labeled as Group 1 in Table 3. We chose this group as a focus group based on the level of changes in reasoning throughout the activities and grade diversity. Indeed, this group showed the most drastic changes in reasoning during the $\varepsilon$-strip activity.

\section{Prior to Episode 1 (Day 7): Students' Primary Intuition About Convergence}

Before the $\varepsilon$-strip activity, the instructor handed out the set of seven sequences in Table 2 and asked students to discuss in groups to evaluate if each of the sequences is a convergent sequence, determine the limit of the sequence if the sequence is a convergent sequence, and create one single criterion to determine the convergence of a sequence. By the meaning of one criterion for the convergence of a sequence, the instructor added a comment that the criterion must be applicable to any sequence so as to determine its convergence or divergence. Since the $\varepsilon-N$ definition was not yet exposed to the students in the course or from other mathematics course taken prior to the IRA course, the students would have developed their intuitive understanding of the convergence of a sequence independently of any systematic instruction of the $\varepsilon-N$ definition. Hence, we considered the students' criteria for convergent sequences at this point as observable evidence of their primary intuition.

All three groups of students in the class correctly determined the convergence or divergence of each sequence in Table 2. In particular, they were aware of the uniqueness of the limit value for a convergent sequence. On the other hand, they were struggling with creating one single criterion for the convergence of a sequence. They tended to consider only the sequences given in Table 2 rather than considering an arbitrary sequence. However, during the whole-class discussion led by the instructor after the group discussion, each group of students presented a single statement for their group's criterion as follows: A sequence is convergent if 
- For all inputs, the output gets closer and closer to a single number. (Group 1)

- The sequence approaches a single number as $n$ gets large and approaches infinity. (Group 2)

- The sequence approaches a single number as $n$ approaches infinity. (Group 3)

It was noted that all three groups used the phrase "approach a single number" or the phrase "gets closer and closer to a single number" in their criteria for the convergence of a sequence. For instance, they paid attention to the behavior of the sequence $\{1 / n\}$ that approaches 0 as $n$ gets larger, and determined that the sequence $\{1 / n\}$ converges to 0 . We identified these students' primary intuition about convergence as "approaching a single number" based on their explanations and the sequences used in their explanations: A sequence is a convergent sequence if the sequence approaches a single number.

The instructor then raised an issue with the ambiguity of the meaning of the word "approaching a single number" in their criteria. At the instructor's request to interpret what they meant by a sequence approaching a single number, Ryan in Group 1 explained that it represented a sequence strictly decreasing or strictly increasing towards a real number. However, the class soon realized that some sequences are not strictly decreasing or strictly increasing towards 0 but converging to 0 , such as a constant sequence $\{0\}$ or an oscillating sequence $\left\{(-1)^{n} / n\right\}$; in addition, the sequence $\{1 / n\}$ strictly decreases towards -0.1 but does not converge to -0.1 . The class then agreed that "a more precise expression than approaching a single number" would be needed as a criterion for the convergence of a sequence. ${ }^{3}$ At this point, the instructor introduced $\varepsilon$-strips to the students and suggested to evaluate the $\varepsilon$-strip definitions $\mathrm{A}$ and B as stated in the "Methodology" section.

\section{Episode 1 (Day 8): the $\varepsilon$-strip Activity}

We organized the first classroom episode, Episode 1, into six phases in terms of its chronological order at the moment when the students in Group 1 started to evaluate the $\varepsilon$-strip definitions $\mathrm{A}$ and $\mathrm{B}$. Each phase in this episode was identified as distinct from the previous one whenever the students' evaluation $\varepsilon$-strip definitions changed. We also identified a new phase whenever the students generated a new statement for a definition of convergent sequences.

Phase 1 (Neither A nor B is correct) The students initially evaluated that none of the $\varepsilon$-strip definitions would correctly describe the convergence of a sequence. For instance, Andy said, "I don't think it's [A] actually the legitimate definition for limit because that would also be the case that if it [a sequence] converges to two different values. In that case, the sequence does not actually have a limit, but that condition [A] would imply that it does." Andy's claim suggests an oscillating divergent sequence to be a counterexample for $\varepsilon$-strip definition $\mathrm{A}$ because such a sequence satisfies

\footnotetext{
${ }^{3}$ We consider that these students' recognition at this point is similar to that in Swinyard's (2011) study in that students' recognition of the 'necessity' of more articulated expressions for a definition was the first step in reinventing a definition of the limit of a function at a point. In the line of standpoint but in more general contexts, Harel (2008) emphasized such a 'necessity principle' in designing curriculum and instruction.
} 
Condition A although it does not have a limit. Other students in this group agreed with Andy's claim that $\varepsilon$-strip definition $A$ is not correct. They also considered $\varepsilon$-strip definition B as incorrect. For instance, Andy argued, "They [A\& B] wouldn't be true [...] because there are certain cases when a sequence doesn't have a limit but these [A \& B] would still be true." Other students expressed their agreement with Andy. They then tried to find a sequence that would not converge but would satisfy Condition B. However, their attempts to find a counterexample for $\varepsilon$-strip definition B were not successful at this phase.

Phase 2 (B is necessary) Comparing to Phase 1 in which the students' foci were mainly on evaluating the correctness of $\varepsilon$-strip definitions A and B, at Phase 2, they specifically examined whether Condition B is necessary. Such a change occurred as a result of students testing Conditions A and B with the sequence (v) in Table 2. Placing an $\varepsilon$-strip on the graph of the sequence $(v)$ and aligning its center at $y=1$, which is one of the cluster points of the sequence, the students observed that infinitely many points are inside and no points are outside the $\varepsilon$-strip, as illustrated in Fig. 3. However, they did not conclude from this instance that the sequence satisfies Conditions A and B. They rather asked themselves if infinitely many points would be inside and no points be outside the $\varepsilon$-strip no matter which $\varepsilon$-strip they were to use. They then realized that the arbitrariness of $\varepsilon$-strips could allow for testing with narrower $\varepsilon$-strips. They pointed out that when using a narrower $\varepsilon$-strip, infinitely many terms of the sequence $(v)$ are both inside and outside the $\varepsilon$-strip (Fig. 4). The students then concluded that such a divergent sequence does not satisfy Condition B but rather satisfies Condition A. Finally, they contended that Condition B is necessary to explain the divergence of the sequence $(v)$.

Dave: Part B takes care of this. (points at the graph of (v), Fig. 4)

Andy: Mmhmm (affirmative)

Dave: but part A [...] would work because there are infinitely many points in this situation $[(v)]$. So we would need it $[\mathrm{B}]$.

Andy: Yeah, and that's basically what we were coming up with before for why part $\mathrm{A}$ is false.

Dave: Yeah.

Andy: 'cause there are counterexamples to it $[\mathrm{A}]$ like this $[(v)]$.

Dave: Yeah.

It is noted from the transcript above that the students evaluated Condition B as a necessary condition. On the other hand, they did not yet deduce the insufficiency of Condition A from the necessity of Condition B. This indicates that the students conceived the insufficiency of Condition A as a separate issue from the necessity of Condition B. 


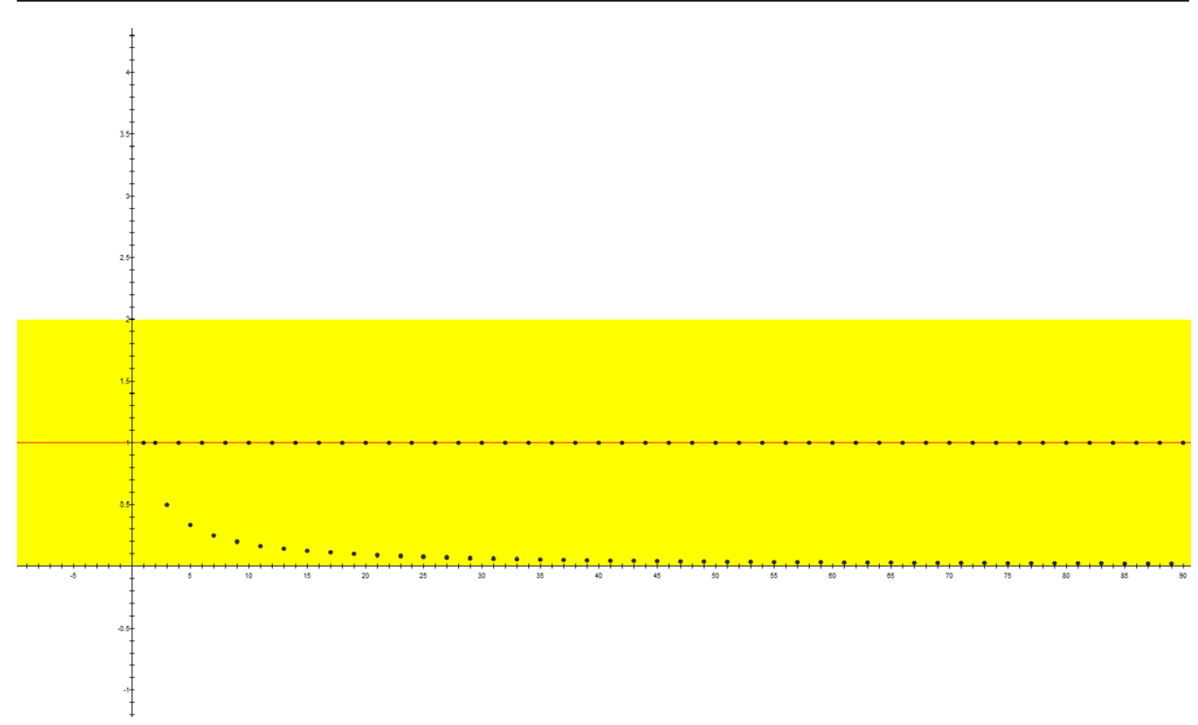

Fig. 3 Only finitely many terms of the sequence $(v)$ are outside an $\varepsilon$-strip

Phase 3 (Both A and B are necessary) At Phase 3, the students evaluated Conditions A and B both to be necessary. The students obviously accepted, without any doubt, the necessity of Condition A. They rather tried to grasp the reason of the necessity of Condition $\mathrm{B}$ as an additional condition to complement the insufficiency of Condition A. For instance, Ryan commented that accepting $\varepsilon$-strip definition A would allow falsely determining 0 to be the limit of the sequence $(v)$ in Table 2. Simultaneously, Dave demonstrated Ryan's idea by placing an $\varepsilon$-strip on the graph of the sequence $(v)$ and aligning its center at 0 . The group then observed that infinitely many points clustered around $y=1$ were outside the $\varepsilon$-strip (Fig. 5) and hence the sequence $(v)$

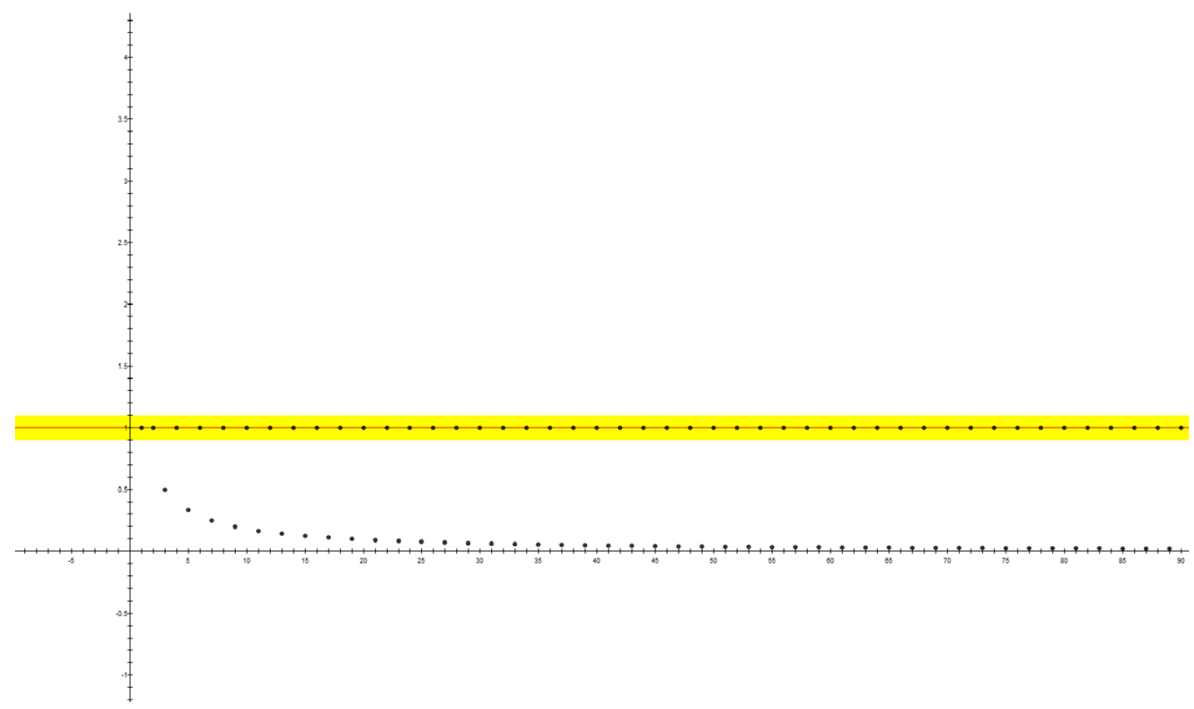

Fig. 4 Infinitely many terms of the sequence $(v)$ are outside a narrower $\varepsilon$-strip 
countered $\varepsilon$-strip definition A. Dave examined another sequence ( $v i$ ) in Table 2 by placing an $\varepsilon$-strip at $y=0$ on top of the graph of the sequence (vi) (Fig. 6). He then pointed out that infinitely many points were inside the $\varepsilon$-strip (Condition A) and only finitely many points were outside the $\varepsilon$-strip (Condition B). This group then concluded Conditions B as necessary to show why 0 is not the limit of the sequence $(v)$ and Condition A as necessary to show why 0 is the limit of the sequence $(v i)$.

Dave: Well, [it] seems you need to consider both [A \& B] to make it work.

Ryan: A and B are necessary conditions to describe a limit. You can't use just one because we were actually looking at this case $[(v)]$ specifically. [...] You can say that in this case $[(v)]$, if you were just to use A, the limit would actually be 0 because there are infinitely many points within that $\varepsilon$-strip.

Dave: (places the center of an $\varepsilon$-strip on the graph of (v) at $y=0$; see Fig. 5).

Ryan: However, clearly this 0 is not the limit of this sequence $[(v)]$. So, we came to a conclusion that A and B were both necessary conditions. Whether it's sufficient or not [is] I think still (inaudible)

Dave: The other we were looking at was this one $[(v i)]$ (places the center of an $\varepsilon$ strip on the graph of (vi) at $y=0$; see Fig. 6). [...] if you use both A and B, it does describe that it $[(v i)]$ converges towards 0 both from the positive and negative

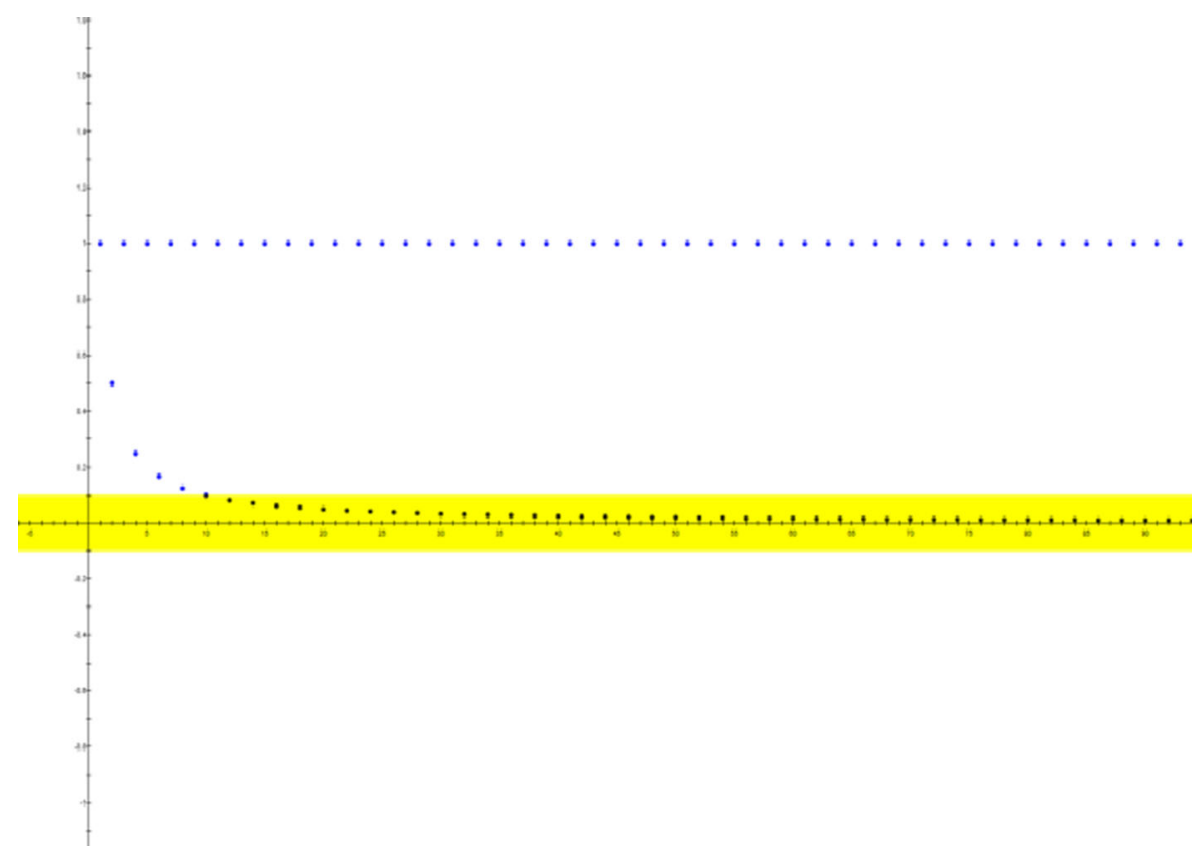

Fig. 5 The graph of (v) where an $\varepsilon$-strip is centered at $y=0$ 


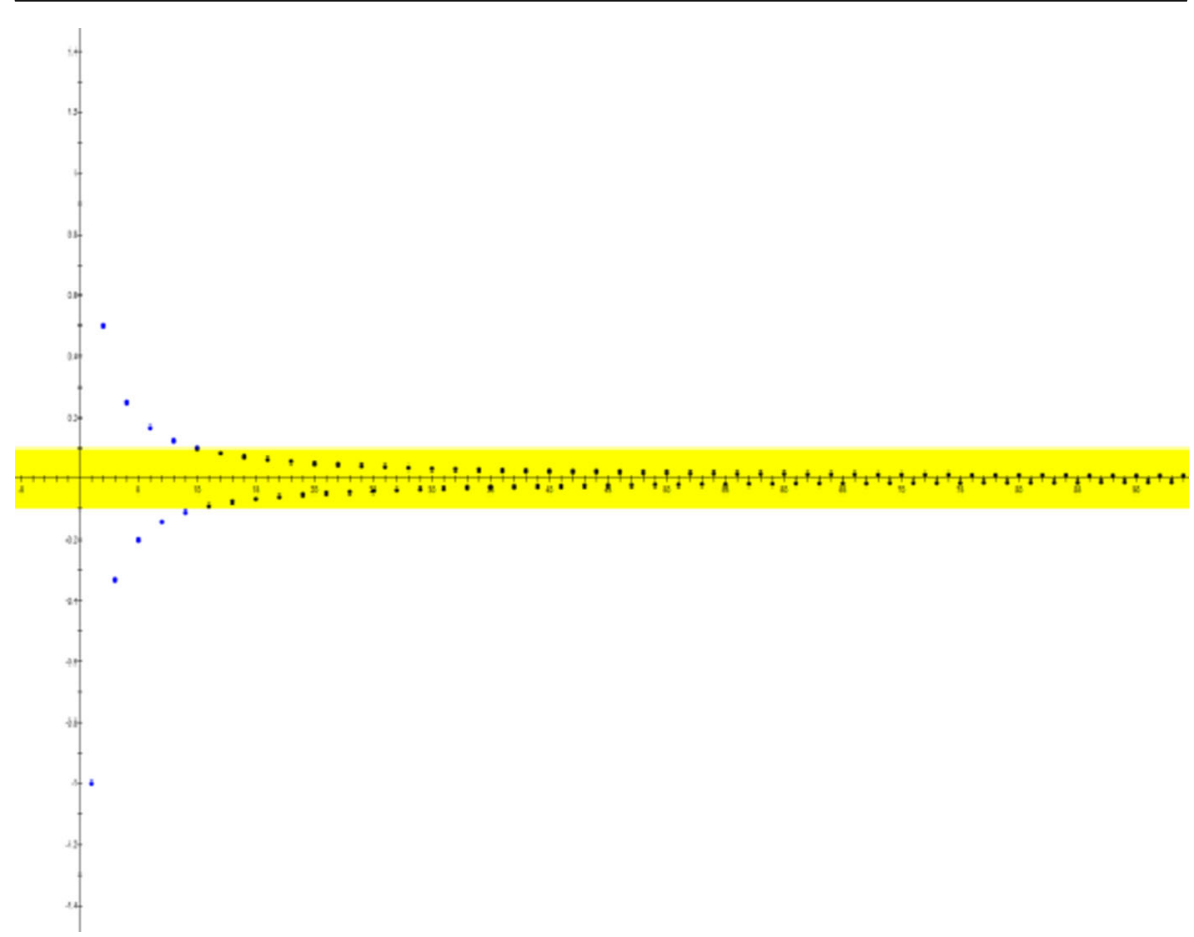

Fig. 6 The graph of ( $v i)$ where an $\varepsilon$-strip is centered at $y=0$

side (flips his palm). [...] you can find infinitely many values and then there is also finite values up here (points at the outside the $\varepsilon$-strip), [...] that follows the definition $[\mathrm{A}+\mathrm{B}]$.

We denote the group's new condition as Condition $\mathrm{A}+\mathrm{B}$ in this paper, which states, "for any $\varepsilon$-strip, infinitely many points are inside the $\varepsilon$-strip and only finitely many points are outside the $\varepsilon$-strip." Phase 3 was the first phase that this group constructed a new statement for a definition of the convergence of a sequence. The students at this phase considered $\varepsilon$-strip definition $\mathrm{B}$ to be still incorrect although they understood that Condition $\mathrm{B}$ is needed to define the convergence of a sequence. It is also indicative that the students at this phase were not yet aware that Condition A could be deduced from Condition B. Up until then, they seemed to consider the insufficiency of Condition A as a separate issue from the necessity of Condition B.

Phase 4 (Neither A nor B is sufficient) At Phase 4, the students evaluated Condition A or B alone to be insufficient. The students' evaluation was observed while they sought an answer to Andy's question: "Is there a situation where considering only $B$ would cause a problem? Because here considering only A causes a problem, do we have the opposite situation or anything?" Responding to Andy's question, Ryan said, "A and B are both necessary. We can't just have one or the other." He explained why Condition A is insufficient by referring to sequences with multiple cluster points, e.g., (v) and (vii), which satisfy Condition A but do not converge. He also thought Condition 
$\mathrm{B}$ is insufficient and expected to find a sequence that satisfies Condition B but does not converge:

Ryan: I'm just really, I really feel and I have no way, like, my gut says that there is a counter example to part B alone. But I cannot, for the life of me, think of one. [...] If you would just take B, part B actually rejects that there is a limit to this problem $[(v)]$. So this is actually an example where B works. [...] The problem that you would have to [do is] to create a counterexample for B. [...] You would need a $L$-value [that is not a limit of the sequence] for which there were a finite number of points outside the strip.

Phase 4 is distinct from the previous phases in the sense that the students considered the insufficiency of Condition A and the insufficiency of Condition B. They verified the insufficiency of Condition A by providing the sequence (v). Similarly, they also tried to find a sequence to justify the insufficiency of Condition B. In fact, the students' attempts to find such a sequence were not successful. However, at this phase, they continued believing that Condition B is insufficient.

Phase 5 (B MAY be Sufficient, so A MAY not be Necessary) At Phase 5, the students started to doubt the necessity of Condition A whereas at Phase 4, they considered Condition A to be necessary. Such a change in the students' evaluation was raised, much to our surprise, in the process of searching for evidence of the insufficiency of Condition B. Ryan predicted that a counterexample to $\varepsilon$-strip definition $B$ should certainly exist because otherwise the non-existence of such counterexamples is against their previous claim that both Conditions A and B are needed.

Ryan: The problem that I am having is that if we cannot find a counterexample for B on its own, then A is [not necessary], then you don't need A and B. You can just use B.

Andy: Mmhmm (affirmative)

Ryan: Um...so, that's just why I think we need a counterexample for B.

Andy: Yeah.

Other students agreed with Ryan for the reason that they had to find out a counterexample for $\varepsilon$-strip definition B. Ryan was then afraid that if they cannot find any counterexample for $\varepsilon$-strip definition $\mathrm{B}$, they would have to evaluate Condition $\mathrm{A}$ as unnecessary. In response, Dave suggested that Condition A might be unnecessary and Condition B alone might be sufficient.

Dave: (to Ryan) You made the comment, and I think this is a good comment. If part B, if we can't disprove part B, then part B is the only one that we need -

Andy \& Ryan: Mmhmm (affirmative) 
Dave: And in all honesty the more I think about (reads $\varepsilon$-strip definition $B$ ), to me it seems like B is the definition we should be using in this situation [...] I'm on the opinion that B works for this. But I know you got your feeling [...] that this [B] doesn't work. But if the four of us have all put our minds to trying to think of it.

Ryan: Yeah.

Dave: And I would say right, and I would throw out there right now. But until we come up with one [counterexample for B], we should accept this [B] as -

Ryan: Okay, I mean I'm always open to be wrong, so

Andy: Yeah, I agree. B doesn't seem like it should be right. But since we haven't found any evidence that it's false, that's what we should be going with for now. $[\ldots]$

Instructor: So your argument is [that] B itself is good enough as a description for the limit of a sequence?

Dave: Only because we cannot find a counterexample. Okay? (laughs)

It was noted that at this phase, the students began to think that Condition B might be sufficient. However, they still apprehended the possibility of a counterexample for $\varepsilon$ strip definition B. Hence they decided to accept $\varepsilon$-strip definition B temporarily until they could find a counterexample for $\varepsilon$-strip definition B. Phase 5 also captures a crucial stage in the students' evaluation in the sense that it was the first phase that the students perceived Condition B may be sufficient. Furthermore, although the students had already generated Condition $\mathrm{A}+\mathrm{B}$ as a proper condition for the convergence of a sequence, they continued trying to draw a more concise condition in which process they paid more attention to $\varepsilon$-strip definition $\mathrm{B}$.

Phase 6 (B is sufficient so $\mathbf{A}$ is not necessary) At Phase 6 , the students finally ascertained that Condition $\mathrm{B}$ is sufficient and so Condition $\mathrm{A}$ is unnecessary. The students' assurance occurred as they made an implication as follows: If only finitely many terms of the sequence are outside an E-strip, then infinitely many terms of the sequence are inside the $\varepsilon$-strip. Assenting to Dave, this group concluded that Condition $\mathrm{A}$ is not needed any longer.

Dave: Well, if you have a sequence that is infinitely long, [...] there is an area (moves his hand to point to the left) where one part of it is finite, that would imply that the other area (moves his hand to the right) would be infinite 'cause you still have an infinite set and infinitely plus a finite value is still infinite. [...] That's why I feel that B is concise enough for the situation. We don't need to say that there are infinite set inside if the entire sequence we're using is infinite.

Findings from Phase 6 are significant in the students' evaluation. First of all, the students' speculation changed to their conviction that Condition A should be 
unnecessary. In addition, their speculation changed to their conviction of the sufficiency of Condition B. The students' proper understanding of the implication structure between the conditions $\mathrm{A}$ and $\mathrm{B}$ played a crucial role in assuring $\varepsilon$-strip definition $\mathrm{B}$ as a definition of convergent sequences. Another particularly interesting point at this phase is that the students were reasoning about Conditions $\mathrm{A}$ and $\mathrm{B}$ over all sequences rather than reasoning about these conditions exhibited by the particular examples given in Table 2. This result indicates that the students reasoned that for any sequence, if it satisfied Condition B, then it satisfied Condition A; and hence they convinced themselves that $\varepsilon$-strip definition $\mathrm{B}$ is a definition of the convergence of a sequence.

\section{Episode 2 (Day 9): the $\varepsilon-N$ Definition}

After the $\varepsilon$-strip activity, the instructor presented the $\varepsilon-N$ definition to the class. It was the first time in the IRA course that the students were exposed to the rigorous definition of convergence.

Students' Comparison of the $\varepsilon-N$ Definition with $\varepsilon$-strip Definitions The instructor asked the students to think individually for about 90 seconds, and then to discuss, in groups, whether or not the $\varepsilon-N$ definition is equivalent to $\varepsilon$-strip definition $\mathrm{A}$ or $\varepsilon$-strip definition B. The following transcript captures the students' comprehension of the $\varepsilon$ $N$ definition right after their individual exploration.

Mary: I can see how it [the $\varepsilon-N$ definition] relates to the A part, but not [the $\mathrm{B}$ part]. I'm trying to figure out exactly how it [the $\varepsilon-N$ definition] relates to B. [...] If you like put them [Conditions A and B] together, there's still infinite or something. (silence) Hold on. (silence, deep breath) I don't know. I'm still trying to absorb that [the $\varepsilon-N$ definition].

Andy: They seem fairly similar except that $[\varepsilon-N]$ definition makes use of $N$ and basically only talks about values that pass that $[N]$. (silence) If they're talking about limit and things approaching infinity, it shouldn't really matter. (silence) I'm not sure that they're logically equivalent.

Ryan: I sorta took the approach of unpacking the $[\varepsilon-N]$ definition of a convergent sequence. I sorta unpacked that (points at " $\left|a_{n}-L\right|<\varepsilon$ " in the $\varepsilon-N$ definition) according to our previous sort of theorems in terms of inequalities. ${ }^{4}$ And you sorta come to the conclusion similar to A where B comes in I noticed is the large $N$ basically. So by defining that large $N$, we're saying that there is some numbers that are smaller than $N$ which are outside of that $\varepsilon$-strip. That's how I thought of it.

Dave: Yeah. [...] It [the $\varepsilon-N$ definition] seems very similar to B in that 1 to the large $N$ is finite amount whereas anything greater than large $N$ is infinite which fits what we were discussing about finite and infinite earlier. $[\ldots]$ and then also

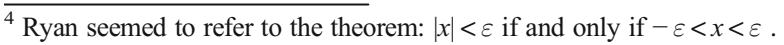


$\left|a_{n}-L\right|$ which is what we did, we've already done that with what we were doing earlier.

Andy: If $L$ really is the limit, then the value $\left[\left|a_{n}-L\right|\right]$ should approach 0 .

As shown in the transcript above, Mary and Andy did not associate the $\varepsilon-N$ definition with $\varepsilon$-strip definition B. Mary instead related the $\varepsilon$ - $N$ definition to $\varepsilon$-strip definition A by still focusing on infinitely many points inside $\varepsilon$-strips. Andy tended to interpret the limit of a sequence as a value that the sequence approaches. Indeed, Mary and Andy did not make use of $\varepsilon$ and $N$ when interpreting the $\varepsilon-N$ definition. Andy noticed the appearance of $N$ in the $\varepsilon-N$ definition, but rather insisted that $N$ is not necessary in defining the limit of a sequence. These students' explanations hence indicate that the $\varepsilon-N$ definition was still counter-intuitive to them, and they relied heavily on their primary intuition. On the other hand, Ryan and Dave perceived the equivalence between the $\varepsilon-N$ definition and $\varepsilon$-strip definition $\mathrm{B}$. They also grasped the meaning of $\varepsilon$ and $N$ in the $\varepsilon-N$ definition by referring to what they were doing earlier during the $\varepsilon$-strip activity. These students' use of $\varepsilon$-strips, with the graphs of given sequences $(i)$ and (ii) in Table 2 , is indicative of them referring to the $\varepsilon$-strip activity. In particular, they related $N$ to a finite number of points outside an $\varepsilon$-strip, and $a_{n}$ in the inequality $\left|a_{n}-L\right|<\varepsilon$ to the infinitely many points inside the $\varepsilon$-strip. This result indicates that the $\varepsilon-N$ definition was not counter-intuitive to Ryan and Dave. Furthermore, their secondary intuition developed via the $\varepsilon$-strip activity played a substantial role in their understanding of the meaning of $\varepsilon$ and $N$ in the $\varepsilon-N$ definition.

Students' Understanding of the Relationship Between $\varepsilon$ and $N$ in the $\varepsilon-N$ Definition For the next five minutes, this group focused more on the relationship between $\varepsilon$ and $N$ in the $\varepsilon-N$ definition. First of all, the students noticed that the value of $N$ is determined based on the value of $\varepsilon$. To illustrate such a relationship, Dave placed an $\varepsilon$-strip on top of a graph of the sequence (ii) in Table 2, aligning its center at $y=1 / 10$ (see Fig. 5). The students in the group then observed that all terms after the 8 th term of the sequence were contained in the $\varepsilon$-strip.

Dave: In this situation, if $N$ would be 8 , then you start getting the situation where (moves an index finger to trace the points outside the $\varepsilon$-strip) this is finite, (moves the index finger to trace the points inside the $\varepsilon$-strip) this is infinite, and any value of $a_{n}$ minus that [1/10] will be less than the width of $\varepsilon$.

Andy: Well, in a situation like this [(ii)], where $a_{n}$ actually does approach a specific value [1/10] and it stays at after a certain point, then you can pin down a specific $N$, which I guess is like 8 or 9 here. But if it was like the $1 / n$ case, then what can we call $N$ ?

Ryan: You can actually, yeah, $N$ is sort of arbitrary.

Dave: Yeah, you can say that there's still a large $N$ where $\left|a_{n}-L\right|$ is smaller than $\varepsilon$, and that fits for each of these situations. [...] It $[N]$ could be anything because you 
could have, since arbitrary values of $\varepsilon$. [As] the value of $\varepsilon$ actually changes, what the $N$ would be? Or, you can still choose any large $N$ and there is still infinitely many values of small $n$ that fit within the $\varepsilon$.

Ryan: Yeah. [...] So, if $\varepsilon=1$, then $N=1$.

It is noted that Dave and Ryan perceived the dependence of $N$ on $\varepsilon$ based on the relationship between the width of an $\varepsilon$-strip and the number of points outside the $\varepsilon$ strip. In the case of the sequence (ii), Dave picked an $\varepsilon$-strip first, and then he determined a value of $N$ to be 8 for the specific $\varepsilon$-strip. He explained how he determined the value of $N$ in the case as follows: For such an $\varepsilon$ value, the number of terms of the sequence that are outside the $\varepsilon$-strip is 8 , which is finite; and the rest of the terms of the sequence after the 8th term, infinitely many terms contained inside the $\varepsilon$ strip, differ from $1 / 10$ within the value of $\varepsilon$ (see Fig. 7). Similarly, Ryan demonstrated his understanding of the dependence $N$ on $\varepsilon$ via the sequence $(i)\left\{\frac{1}{n}\right\}$. He determined that in the case of $\varepsilon=1$, the value of $N$ to be 1 (see Fig. 8).

On the other hand, Andy did not seem to grasp the dependence of $N$ on $\varepsilon$ at this moment. He pointed out that in the case of the sequence (ii), the value of $N$ can be determined because the sequence "stays at" its limit after the 8th or 9th term; however, in the case of the sequence ( $i$ ) $\left\{\frac{1}{n}\right\}$, the value of $N$ cannot be determined because the sequence continues approaching rather than staying at a value. This could imply that Andy considered the value of $N$ in the $\varepsilon-N$ definition as a value that no matter what positive real number $\varepsilon$ is, all terms of the sequence, after the $N$-th term, are inside the $\varepsilon$ strip. In other words, he would conceive the $\varepsilon-N$ definition by reversing the order between $\varepsilon$ and $N$ as follows: "There exists $N$ so that for any positive real number $\varepsilon$, for all $n>N,\left|a_{n}-L\right|<\varepsilon$." Andy's utterance at this point indicates that he was still relying heavily on his primary intuition and could not perceive the dependence of $N$ on $\varepsilon$ in the $\varepsilon-N$ definition.

Arguing against Andy, Dave and Ryan contended that in the case of the sequence $(i)$, the value of $N$ can be determined based on the value of $\varepsilon$. In addition, they pointed out that the value of $N$ does not have to be fixed, but would vary as the value of $\varepsilon$ varies. Dave described that for the specific $\varepsilon$-strip in Fig. 7, the natural number 8 was

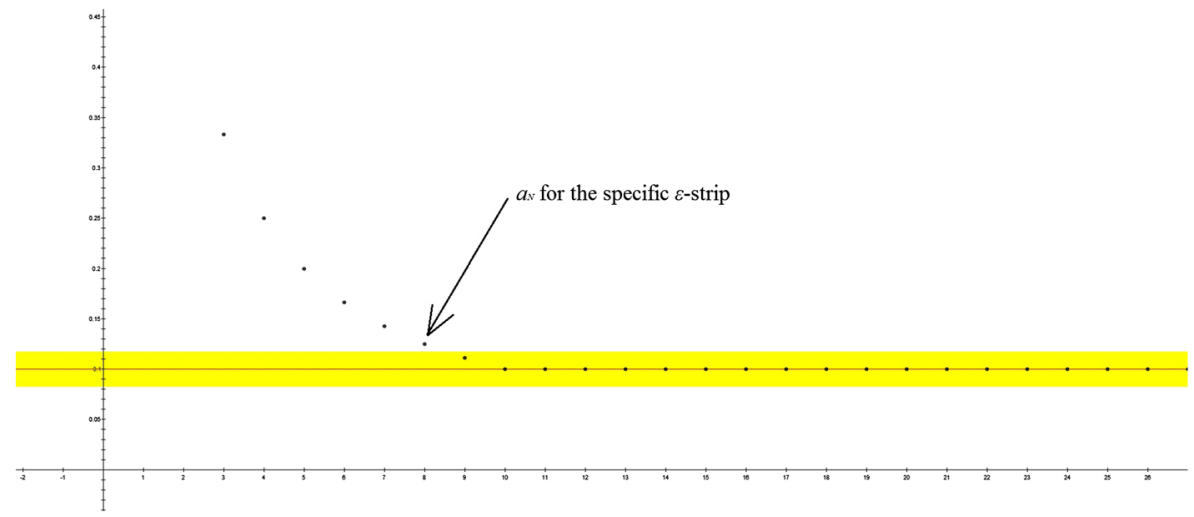

Fig. 7 Dave's interpretation of the dependence of $N$ on $\varepsilon$ with the sequence (ii) in Table 2 


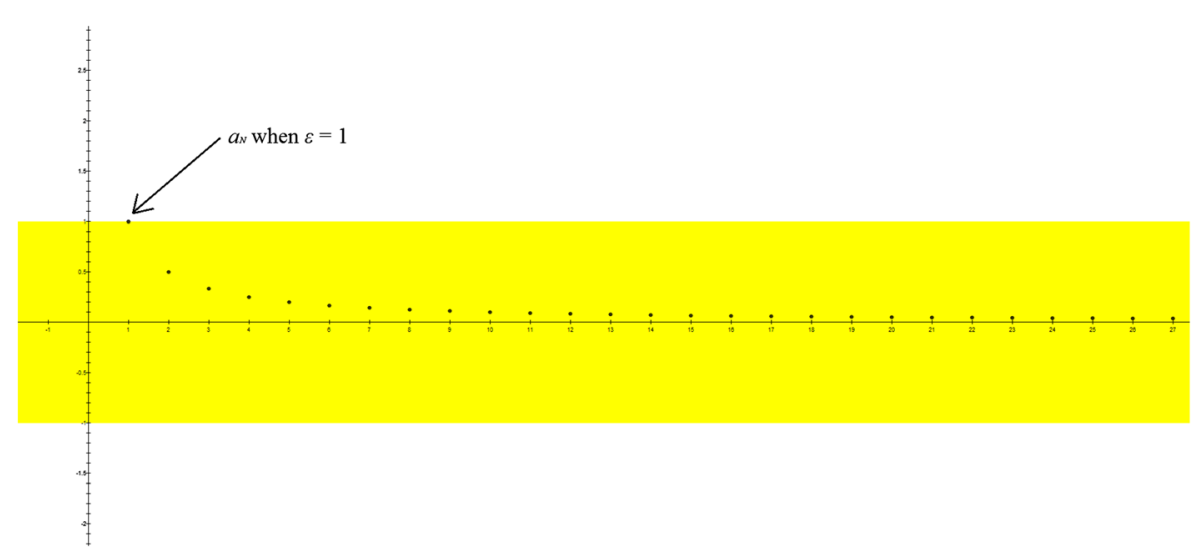

Fig. 8 Ryan's interpretation of the dependence of $N$ on $\varepsilon$ with the sequence $\left\{\frac{1}{n}\right\}$

determined as a value of $N$; however, if a different $\varepsilon$-strip was chosen, a different natural number may be determined for a value of $N$. He pointed out that the value of $N$ will always be determined by the value of $\varepsilon$ no matter which sequence is examined. Here, Dave and Ryan perceived not only the dependence of $N$ on $\varepsilon$ but also the variation of $N$ corresponding to the variation of $\varepsilon$. It is noteworthy that these students used $\varepsilon$-strip definition $\mathrm{B}$ in their utterances, gestures, and diagrams to explain the relationship between $N$ on $\varepsilon$ in the $\varepsilon-N$ definition. Their use of $\varepsilon$-strips, with the graphs of given sequences $(i)$ and (ii) in Table 2, is indicative of them referring to the $\varepsilon$-strip activity. In addition, considering the fact that $\varepsilon$-strip definition $\mathrm{B}$ was not part of the students' primary intuition, their secondary intuition developed via the $\varepsilon$-strip activity played an important role in their understanding of the dependence of $N$ on $\varepsilon$, the variation on the value of $\varepsilon$, and the corresponding variation on the value of $N$. Andy later seemed to grasp such a relationship between $\varepsilon$ and $N$ throughout the group discussion as well as class discussion. In the next episode, Episode 3, we will report Andy's emerging grasp on the relationship between $\varepsilon$ and $N$ when he interpreted the meaning of Cauchy sequences.

Students' Comprehension of the $\varepsilon-N$ Definition The group finally visually represented the $\varepsilon-N$ definition along with an $\varepsilon$-strip (see Fig. 9). Although they drew a specific $\varepsilon$-strip on the diagram, they clarified it to be an arbitrary one. In addition, they often moved hands up and down vertically between the top edge of the $\varepsilon$-strip and its center, considering the distance between them as the value of $\varepsilon$. Once marking $N$ as the entry point to the $\varepsilon$-strip in their diagram, they moved an index finger along the $x$-axis from $N$ to the left to refer to a finite number of points outside the $\varepsilon$-strip. They also moved an index finger from $N$ to the right to refer to the points inside the $\varepsilon$-strip. Dave summarized his group's discussion as follows:

Dave: How I've been viewing it $[\varepsilon]$ is a length of $\varepsilon$-zone. It $[\varepsilon]$ has to be greater than 0 . So for any value of $\varepsilon$, and this $[\varepsilon]$ is just an arbitrary one, we can find a point $N$ such that all values of small $n$ that I remember are naturals after this $[N]$ are contained within this value of $\varepsilon$. So, basically this $\left[a_{n}\right]$ minus the value of $L$ will give you, the absolute value of that $\left[\left|a_{n}-L\right|\right]$ will give you a value less than $\varepsilon$ 


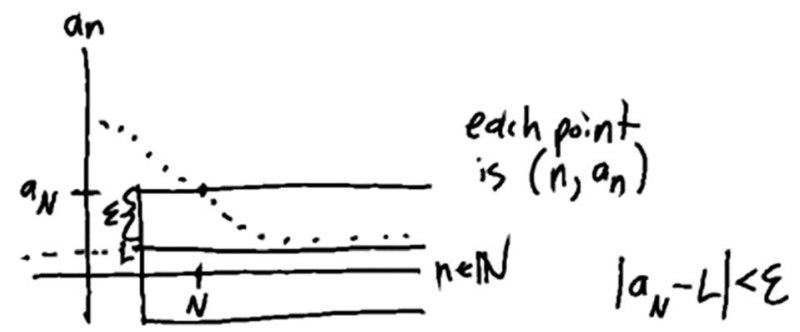

Fig. 9 Andy's diagram in his note as a copy of Dave's diagram on the board

[...] What we find is that we can find a point where all of these points can be counted, and all these can be infinite [...] and all each of the infinite points are contained within the $\varepsilon$-strip. [...] Whatever arbitrary point you choose in here [inside the $\varepsilon$-strip] would be $a_{n}[\ldots]$ as long as $n$ is larger than $N$. I'm just thinking individual dots, but each of these dots will be small $n$. And if you pick any arbitrary value (points to a dot inside the $\varepsilon$-strip), then it will be $a_{n}$.

The students' explanations with their diagrams and gestures indicate that they appropriately interpreted the $\varepsilon-N$ definition via $\varepsilon$-strip definition $\mathrm{B}$ : (1) The symbol $\varepsilon$ in the $\varepsilon-N$ definition is quantified as the half of the width of an arbitrary $\varepsilon$-strip; (2) the absolute value symbol $\left|a_{n}-L\right|$ in the $\varepsilon-N$ definition is quantified as the distance between the value of $a_{n}$ and the value of $L$; (3) the symbol $N$ in the $\varepsilon-N$ definition is associated with the number of points outside the $\varepsilon$-strip; (4) the index $n$ in the quantification "for all $n>N$ " refers to the first coordinates of the infinite number of points $\left(n, a_{n}\right)$ after the finite number of points outside the $\varepsilon$-strip; (5) the term $a_{n}$ in the inequality $\left|a_{n}-L\right|<\varepsilon$ refers to the infinitely many points inside the $\varepsilon$-strip; and (6) the value of $N$ is determined depending on the value of $\varepsilon$.

\section{Episode 3 (Day 14): a Theorem About Convergent Sequences and Cauchy Sequences}

After 2 weeks from the day of Episode 2, the instructor introduced the definition of Cauchy sequences ${ }^{5}$ to the students, and asked them to prove or disprove the statement (*): "Every convergent sequence is a Cauchy sequence." Compared to traditional IRA courses, the organization of the proving task in our inquiry-oriented IRA course could create a more challenging situation to the students. Up until that day, the students had not yet seen the definition of Cauchy sequences or constructed any rigorous proof of a statement about Cauchy sequences. In addition, the truth of the statement $\left(^{*}\right)$ was not given to the students, but the students were rather asked whether or not the statement (*) is true. Such a different organization made the proving task more difficult, compared to traditional IRA courses in which the statement $(*)$ is given as a theorem and its proof is provided by a course instructor (e.g., Knapp and Roh 2008).

\footnotetext{
${ }^{5}$ In this paper we refer to the following statement as the definition of Cauchy sequences: For a set $S \subseteq \mathbf{R}$, a sequence $\left\{a_{n}\right\}_{n=1}^{\infty}$ in $\mathrm{S}$ is a Cauchy sequence if for any $\varepsilon>0$, there exists $N \in \mathbf{N}$ such that for all $n, m>N$, $\left|a_{n}-a_{m}\right|<\varepsilon$.
} 
Students' Understanding of Cauchy Sequences After a few minutes of individual exploration, the students determined the sequence $\left\{\frac{1}{n}\right\}$ to be a Cauchy sequence. Ryan reasoned that the distance between two arbitrary terms of the sequence $\left\{\frac{1}{n}\right\}$ is decreasing. Andy further pointed out that the definition of Cauchy sequences is similar to the $\varepsilon$ $N$ definition, and interpreted a sequence to be a Cauchy sequence if, for a value picked for $\varepsilon$, there is $N$ beyond which the difference between any two terms of the sequence is less than the value of $\varepsilon$. Agreeing with the two students, Dave added a comment that whatever the value of $\varepsilon$ is picked, the value of $N$ can be chosen so that for all values of $n$ and $m$ larger than $N,\left|\frac{1}{n}-\frac{1}{m}\right|$ becomes less than $\varepsilon$.

Ryan: I'm pretty sure that $\{1 / n\}$ is actually a Cauchy sequence in $\mathbf{Q}$ because basically what this [Cauchy definition] is saying is the distance between two $a_{n}$ 's, two arbitrary $a_{n}$ 's, is getting smaller and smaller.

Andy: Yeah. That's [Cauchy definition] actually kind of similar to the limit definition that we had before. Basically the way that I look at this [Cauchy definition] is saying that if you pick some $\varepsilon$ distance, there's going to be a point $N$ and beyond that point, the difference between any two given values will be less than that $\varepsilon$. And since you can let $\varepsilon$ become as small as you want, if you choose something $[\varepsilon]$ that approaches 0 , then the distance [between two terms of the sequence] should approach 0 , which means that you're approaching a particular value.

Dave: Yeah, essentially I was running into the same thing where basically because the value of $\varepsilon$ is actually, whatever the value of $\varepsilon$ you have is, arbitrary. But once you get to know that value of $\varepsilon$, you can choose your $N$. And since you can choose your $N$, all values of $n$ and $m$ are going to be larger than that value of $N$. But since the sequence is getting closer and closer to 0 , your value of $\frac{1}{n}$ and $\frac{1}{m}$ are going to get smaller and smaller, and so the absolute value of the difference is going to end up becoming less than $\varepsilon$.

The transcript above shows that Andy and Dave perceived the value of $\varepsilon$ is arbitrarily chosen, and the value of $N$ is determined depending on the value of $\varepsilon$. They spontaneously understood these ideas from the definition of Cauchy sequences. It is remarkable that the students perceived and utilized the relationship between $\varepsilon$ and $N$ in a short time period of being exposed to the definition of Cauchy sequences. Such an immediate perception was probably because they applied their secondary intuition to the case of Cauchy sequence as they had already done in internalizing the relationship between $\varepsilon$ and $N$ in the case of the $\varepsilon-N$ definition.

The students also used $\varepsilon$-strips in order to make sense of the inequality " $\left|a_{n}-a_{m}\right|$ $<\varepsilon$ " in the definition of Cauchy sequences. Dave drew a pair of horizontal lines on the graph of the sequence $\left\{\frac{1}{n}\right\}$, and called it an $\varepsilon$-strip. He then marked $\varepsilon$ for the distance between the horizontal lines. For the $\varepsilon$-strip, he determined a specific index for $N$ where a finite number of terms of the sequence were outside the $\varepsilon$-strip and all other terms were inside the $\varepsilon$-strip. He then labeled two points inside the $\varepsilon$-strip as $a_{n}$ and $a_{m}$ 
where $n>m>N$. His diagram (Fig. 10) indicates that he perceived that the difference between $a_{n}$ and $a_{m}$ was smaller than the width of the $\varepsilon$-strip.

Dave: So let's say we choose $\varepsilon$ here, right. Then we have that's $N$.

Ryan: Right.

Dave: Now we have three situations, right? Umm, we have where $n$ can be equal to $m$, we have $n>m$, and we have $n<m$, right?

Ryan: Yeah.

Dave: (picks two dots inside the $\varepsilon$-strip and labels them as $a_{m}$ and $a_{n}$, respectively; see Fig. 9) So, this $\left[a_{n}\right]$ minus this $\left[a_{m}\right]$, absolute value, right?

Ryan: Right, so you're basically measuring the distance between those two.

Dave: [...] You're just measuring this length $\left[\left|a_{n}-a_{m}\right|\right]$ right here and then comparing it to this length $[\varepsilon]$ right here (Fig. 10).

According to the transcript above and Fig. 10, the students referred to an $\varepsilon$-strip to the value of $\varepsilon$, and determined the value of $N$ corresponding to the chosen $\varepsilon$-strip. Based on such a visual representation, they also chose $a_{n}$ and $a_{m}$ from the inside of the $\varepsilon$-strip, and compared the difference between two terms $\left|a_{n}-a_{m}\right|$ with the width of the $\varepsilon$-strip. Although such a visual image may not be a precise one, it was enough for the students to immediately determine that the sequence $\left\{\frac{1}{n}\right\}$ is a Cauchy sequence.

An Issue Raised and its Resolution Made in Proving the Statement (*) It was observed that the students unpacked the statement (*) as a conditional statement "If a sequence is a convergent sequence, then the sequence is a Cauchy sequence." They also framed their proof by assuming the hypothesis of the conditional (a sequence is a convergent sequence), and setting up the conclusion of the conditional (the sequence is a Cauchy sequence) as the goal of their proof. Up until then, the students reasoned based on monotone convergent sequences. However, as they took into account

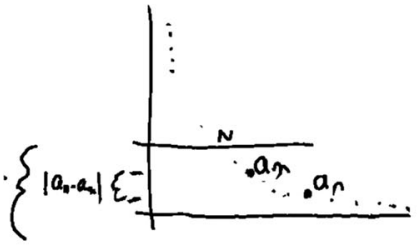

$$
\begin{aligned}
& n=m \\
& n<m \\
& n>m
\end{aligned}
$$$$
3 \text { situotions }
$$

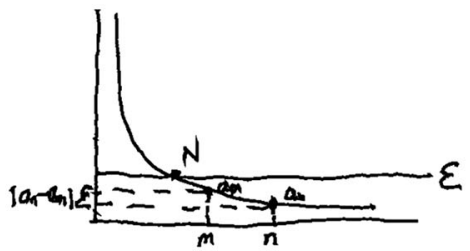

$$
\begin{aligned}
& \text { For } n>m, \quad\left|a_{n}-a_{m}\right|=\left|\frac{1}{n}-\frac{1}{m}\right| \\
& =d_{i s t a n c e} \text { baterean } \frac{1}{n} \text { and } \\
& \text { thei is rompared to Elengthin }
\end{aligned}
$$

Fig. 10 Dave's digram: The sequence $\left\{\frac{1}{n}\right\}$ is a Cauchy sequence. 
oscillating convergent sequences, they raised an issue in proving the statement. Dave demonstrated the issue by sketching an $\varepsilon$-strip where its center was aligned at the limit of an oscillating convergent sequence. He was concerned that the distance between any two points inside the $\varepsilon$-strip must not be less than $\varepsilon$. For the reason, Dave thought that the oscillating convergent sequence might be not a Cauchy sequence.

Dave: The situation that I ran into from looking at is [...] when something converges from the top and from the bottom. (sketches a monotone decreasing sequence) It's an alternating sequence that converges from the top and the bottom. (draws an $\varepsilon$-strip over the graph of the monotone decreasing sequence) So, you have an $\varepsilon$ value here. (draws a line at the center of the $\varepsilon$-strip) This is our $L$ [limit]. (points at one point on the top edge of the $\varepsilon$-strip, the third dot from the left, Fig. 11) So, that $[N]$ is 3 .

What you run into is that if the sequence were convergent and it was just one sequence coming from the top going towards the bottom, then the difference between the two, is gonna be less than the $\varepsilon$ because all values after that point are less than the $\varepsilon$, right? But, as you get into this situation where it's coming from the top and from the bottom (draws some more dots below the line $y=L$ where the new dots form a monotone increasing sequence, converging to L, Fig. 11). There's something that I can't prove it right now and I would need to think it through more. But I'm getting the feeling that this convergent sequence does not necessarily imply a Cauchy sequence.

Ryan also faced with the same problem when attempting to construct a proof of the statement (*) (see Fig. 12). He generated two inequalities $\left|a_{n}-L\right|<\varepsilon$ and $\left|a_{m}-L\right|<\varepsilon$ from the assumptions that the sequence is convergent to $L$ and both $n$ and $m$ are greater than $N$. He said, "we can put those together somehow with the triangle inequality," indicating that he expected the sum of the two absolute values $\left|a_{n}-L\right|$ and $\left|a_{m}-L\right|$ to be less than $\varepsilon$. However, this sum entailed $2 \varepsilon$ instead of $\varepsilon$, which was considered as a challenging situation to the students in proving the statement $(*)$.

Dave and Ryan's challenge is mainly due to their use of the same symbol $N$ for two possibly different values in the $\varepsilon-N$ definition and the definition of Cauchy sequences.

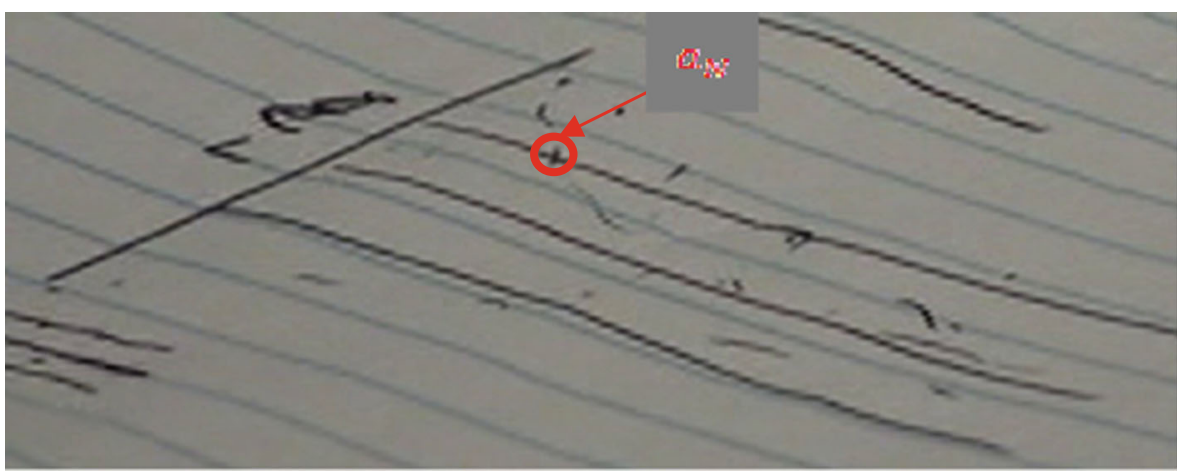

Fig. 11 The issue raised by Dave with $\left|a_{m}-a_{n}\right|<2 \varepsilon$ 


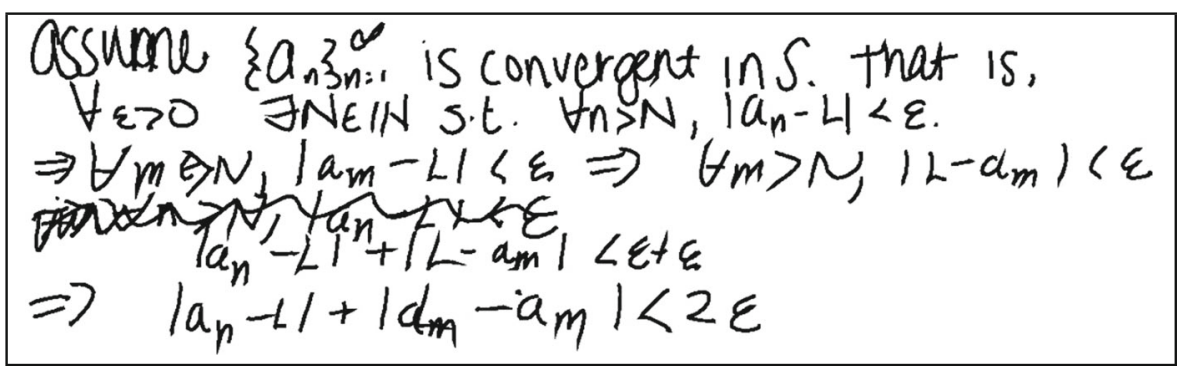

Fig. 12 Ryan's scratch work addressing the issue with $\left|a_{m}-a_{n}\right|<2 \varepsilon$

It is likely that the students treated the symbol $N$ in the $\varepsilon-N$ definition was not a placeholder but a specific value that carried its meaning beyond the $\varepsilon-N$ definition because the symbol $N$ was also used in the definition of Cauchy sequences. On the other hand, Andy noticed that the value of $N$ in the definition of Cauchy sequences must not be the same as the value of $N$ in the $\varepsilon-N$ definition. He also pointed out that it is enough to choose a proper value for $N$. Dave thereupon realized that a new value for $N$ can be chosen further from the value of $N$ chosen for the $\varepsilon-N$ definition. He moved his hand along the $x$-axis further to the right in order to mark a new value of $N$ where the difference between any two terms after the new $N$-th term is less than $\varepsilon$ (see Fig. 13).

Andy: Umm, in this situation, it wouldn't work necessarily to pick $N$ right where $\varepsilon[$-strip] intersects the sequence because the distance between these two points $\left[\left|a_{n}-a_{m}\right|\right]$ is greater than $\varepsilon$. But, you just have to show that there exists an $N$. So if you just chose one further down the line, it would still work.

Dave: So, choose an $N$ down here? (moves his hand along the $x$-axis further to the right) Yeah, okay.

Andy: Umm-hmm (Affirmative)

Dave: So then, I guess the important thing to denote for is that $N$ in a convergence and $N$ in a Cauchy sequence are not always the same.

Ryan: Right.

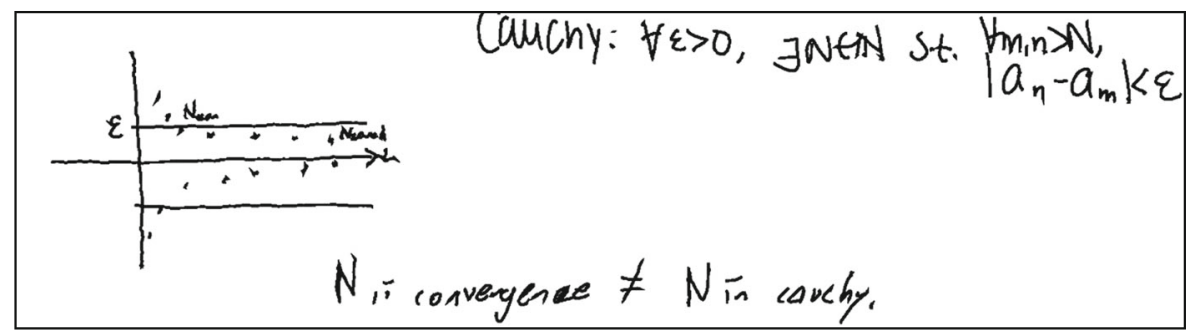

Fig. 13 The resolution of the issue by choosing $N_{\text {cauchy }}$ further down to the right from $N_{\text {con }}$ 
Dave: So we would need to denote $N_{\text {con }}$ and $N_{\text {cauchy }}$

The students only needed about twenty minutes to raise and resolve such an issue with proving the statement $(*)$. In particular, the visual representation of the situation via an $\varepsilon$-strip gave the students an opportunity to reflect on their reasoning in choosing a relevant value for $N$. As a consequence, the students perceived that the value of $N$ could be chosen differently in showing a convergent sequence to be a Cauchy sequence, even if the same symbol $N$ was used in the definition of Cauchy sequences as well as in the $\varepsilon-N$ definition. In fact, by moving a finger further to the right horizontally, they chose a greater value $N_{\text {cauchy }}$ than $N_{\text {con }}$.

\section{Discussion and Concluding Remarks}

This study aimed to explore how an IRA course can be designed to bridge a gap between students' intuitive understanding and mathematical rigor. In this paper, we described an example of a task, the $\varepsilon$-strip activity, and reported how students developed their secondary intuition of the convergence of a sequence through the activity. The results of this study show that the $\varepsilon$-strip activity played a crucial role in bridging a gap between students' intuition and mathematical rigor. Episode 1 revealed that via the $\varepsilon$-strip activity, the students developed their secondary intuition of convergence by internalizing $\varepsilon$-strip definition B. Episode 2 demonstrated that the students conceptualized the $\varepsilon-N$ definition by employing their secondary intuition developed through the activity. Finally, in Episode 3, we illustrated that many students properly reasoned to prove the statement $(*)$ about convergent sequences by utilizing their secondary intuition developed through the $\varepsilon$-strip activity.

\section{How Did Students Develop Secondary Intuition About Convergence?}

At the beginning of the $\varepsilon$-strip activity in Episode 1, the students evaluated that neither $\varepsilon$-strip definition A nor $\varepsilon$-strip definition B is correct. However, at the end of the $\varepsilon$-strip activity, they properly evaluated that $\varepsilon$-strip definition $\mathrm{A}$ is incorrect and accepted $\varepsilon$ strip definition B. In fact, there were some shifts in the students' evaluations until they concluded an appropriate definition of convergent sequences. We summarize these key moments below and describe how such shifts occurred.

Condition A is Insufficient Most students in the group first realized that although the sequences $(v)$ and (vii) with multiple cluster points are not convergent, these sequences satisfy Condition A. As a consequence, they evaluated Condition A to be insufficient. Such a moment occurred as we illustrated at Phase 4 in Episode 1. This result indicates that recalling divergent sequences with multiple cluster points plays a pivotal role in the students' evaluation of the insufficiency of Condition A.

Condition A is Unnecessary The students in the group determined $\varepsilon$-strip definition A to be incorrect as a definition of convergent sequences. In particular, they successfully generated the following implication: If only a finite number of terms of a sequence are 
outside an $\varepsilon$-strip, then infinitely many terms of the sequence are inside the $\varepsilon$-strip. Based on this implication, they evaluated correctly that they did not need to include Condition $\mathrm{A}$ in a definition of the convergence of a sequence because it was already entailed by Condition B.

Condition B is Necessary Initially, all students in the group falsely determined $\varepsilon$-strip definition $\mathrm{B}$ as an incorrect one. However, they later realized the necessity of Condition B. Such a shift occurred as they examined sequences with multiple cluster points. It is noteworthy that students did not recognize the necessity of Condition B when they used such divergent sequences to verify their evaluation of the insufficiency of Condition A. This is an example of a situation where the students considered the examination of the necessity of Condition B as distinct from the examination of the insufficiency of Condition A. We had illustrated such a moment at Phase 2 of Episode 1. The sequences with multiple cluster points again served as pivotal examples in this evaluation shift. In particular, the sequence $(v)$ in Table 2 played a crucial role in helping students accept Condition $\mathrm{B}$ as a necessary condition.

Condition B is Sufficient Students did not immediately accept Condition B as a sufficient condition when they accepted Condition B as a necessary condition. Rather, the students determined Condition B to be sufficient after they accepted the unnecessity of Condition A. Indeed, they determined Condition A + B as a sufficient condition. However, they later reasoned that for any sequence, if the sequence satisfied Condition $\mathrm{B}$, then the sequence satisfied Condition A and they finally accepted Condition B as a sufficient condition.

\section{How Did the Students' Secondary Intuition Play a Role in Understanding the $\varepsilon-N$ Definition?}

In Episode 2, we have reported that the students successfully conceptualized the $\varepsilon-N$ definition by comparing it with $\varepsilon$-strip definition $\mathrm{B}$. They spent about 20 minutes to discuss the equivalence between the $\varepsilon-N$ definition and $\varepsilon$-strip definition $\mathrm{B}$. Initially, a couple of students expressed their difficulty with understanding the $\varepsilon-N$ definition. In particular, they were questioning what $\varepsilon$ and $N$ stand for in the $\varepsilon-N$ definition, why $\varepsilon$ and $N$ are necessary in defining the convergence of a sequence, and how the values of $\varepsilon$ and $N$ are determined. However, as soon as they recalled the $\varepsilon$-strip activity, they resolved these questions. In particular, they interpreted the meaning of the convergence of a sequence via $\varepsilon$-strip definition B instead of from the idea of approaching. Thus, the evidence suggests that their secondary intuition, developed throughout the $\varepsilon$-strip activity, played a substantial role in their conceptualization of the $\varepsilon-N$ definition.

Why is $\varepsilon$ Necessary? The students interpreted the symbol $\varepsilon$ in the $\varepsilon-N$ definition as the half of the width of an $\varepsilon$-strip. They also distinguished the value of $\varepsilon$ from the distance between a term $a_{n}$ and $L$. They rather conceived $\varepsilon$ as an error bound to be compared with any distance between $a_{n}$ and $L$. Furthermore, they understood that $\varepsilon$ in the $\varepsilon-N$ definition would be arbitrarily chosen as an $\varepsilon$-strip in $\varepsilon$-strip definitions would be arbitrarily chosen. Considering that prior to the $\varepsilon$-strip activity, the students described 
the convergence of a sequence as the terms of the sequence approach, or get closer to, a certain value, their actions of decreasing the values of $\varepsilon$ indicate a meaningful shift in their understanding of convergence.

Why is $N$ Necessary? The students immediately perceived the necessity of $N$ by associating it with $\varepsilon$-strip definition $\mathrm{B}$. In particular, they referred to $N$ as a fixed index, distinguishing it from other indices $n$ 's, and associated the value of $N$ with the finite number of points outside an $\varepsilon$-strip. They also considered $a_{n}$ in the inequality $\left|a_{n}-L\right|$ $<\varepsilon$ as a representation for the infinitely many terms of the sequence inside an $\varepsilon$-strip.

Dynamic Images of the Dependence of $\boldsymbol{N}$ on $\varepsilon$ The students understood the dependence of $N$ on $\varepsilon$. Furthermore, they perceived such a dependence in terms of dynamic images where the value of $N$ would change as the value of $\varepsilon$ varies. It is noteworthy that the students' visual representation of the dependence relationship was not limited to a specific case of $\varepsilon$ but applied to smaller ones, in which they took a bigger value of $N$ than the previous one. As a consequence, they visually described the dynamics of varying $\varepsilon$ and $N$ with a specific dependence relationship. These descriptions indicate a significant shift compared with the students' primary intuition in which they considered the convergence of a sequence as the dynamics of the sequence approaching a value. This result indicates that the students' secondary intuition played a crucial role in conceptualizing the convergence of a sequence. We thus regard Episode 2 as evidence of the $\varepsilon$-strip activity bridging a gap between students' intuitive understanding of convergence and the rigorous definition of convergent sequences.

\section{How Did the Students' Secondary Intuition Play a Role in Proving a Theorem Related to Convergence?}

In Episode 3, we reported student challenges encountered when proving the statement (*) and the resolution of the challenges. We also reported that the students properly reasoned to prove the statement $\left(^{*}\right)$ even in the limited time (about 20 minutes) given for the task.

Understanding the Definition of Cauchy Sequences The students interpreted the definition of Cauchy sequences by using $\varepsilon$-strips as follows: The symbol $\varepsilon$ is the width of an $\varepsilon$-strip, and the symbols $a_{n}$ and $a_{m}$ in the inequality $\left|a_{n}-a_{m}\right|<\varepsilon$ are any two points inside the $\varepsilon$-strip. In addition, they also understood $\varepsilon$ as an arbitrary error bound as well as the value of $N$ to be determined depending on the value of $\varepsilon$. In fact, such a relationship between $\varepsilon$ and $N$ was self-evident as they had already internalized it in the case of the $\varepsilon-N$ definition (see Episode 2). Sometimes the students referred to just $\varepsilon$ and $N$ without explicitly recalling an $\varepsilon$-strip and the finite number of points outside the $\varepsilon$ strip, respectively. This result indicates that the students' secondary intuition was still available in understanding the definition of Cauchy sequences.

Proving the Statement (*) The students determined the statement (*) to be true. They also properly structured their proofs of the statement $(*)$ by assuming that a sequence is a convergent sequence, produced a series of valid implications, and set up the 
conclusion that the sequence is a Cauchy sequence. On the other hand, as they examined oscillating convergent sequences, they encountered a difficulty with proving the statement (*). Their challenge was in deducing the inequality $\left|a_{n}-a_{m}\right|<\varepsilon$ as we described it in detail in Episode 3. In particular, they raised a question about how to deduce $\left|a_{n}-a_{m}\right|<\varepsilon$ from $\left|a_{n}-a_{m}\right|<2 \varepsilon$. For instance, Dave examined an oscillating convergent sequence where two points could be chosen from opposite sides of the $\varepsilon$ strip from its center line. He came up with the inequality $\left|a_{n}-a_{m}\right|<2 \varepsilon$ but realized that they could not conclude $\left|a_{n}-a_{m}\right|<\varepsilon$ in this situation. However, the students in his group later realized that when determining if a sequence is a Cauchy sequence, they did not need to use the value of $N$ that was selected for the convergence of a sequence. In particular, a dynamic image of increasing the value of $N$ as the value of $\varepsilon$ decreases helped the students choose a bigger value of $N$ for a Cauchy sequence and hence resolved their issue with concluding $\left|a_{n}-a_{m}\right|<\varepsilon$. This result indicates that the students' secondary intuition about the coordination between $\varepsilon$ and $N$, developed via the $\varepsilon$ - strip activity, also enabled them to prove the statement $(*)$.

\section{Students' Intuitive Understanding as Collective Knowledge Constructed Throughout the IRA Course}

In the "Results" section, we have illustrated a number of situations in which the students gave meanings to the $\varepsilon-N$ definition and the definition of Cauchy sequences. It was noted that the students did not attempt to make sense of specific numerical values for many parts of these definitions, such as $\varepsilon, N, n, a_{n},\left|a_{n}-L\right|$, or $\left|a_{n}-L\right|<\varepsilon$. They rather used $\varepsilon$-strips or produced diagrams reminiscent of the $\varepsilon$-strips in order to conceptualize the relationships among $\varepsilon, N, n$, and $a_{n}$ in these definitions. Therefore, we consider that these students developed their secondary intuition of the $\varepsilon-N$ definition by engaging in the $\varepsilon$-strip activity.

It was often observed that when a student (a speaker) proposed an idea or explained his or her reasoning, other students (non-speakers) in the group were in silence, or at best they were nodding or said simply words such as "yes" to express their consent or affirmation of the speaker's idea. However, when non-speakers used the speaker's idea later in the course, it was evident that such an idea was not only the original speaker's idea but also became the group's shared idea. For instance, in Day 9 (Episode 2) when Dave and Ryan explained the dependence of $N$ on $\varepsilon$ in the $\varepsilon-N$ definition, Andy did not understand such an idea on that day. Later at the same day when Ryan illustrated the dependence relationship with the graph of the monotone convergent sequence ( $i$ ) in Table 2, Andy became silent. However, in Day 14 (Episode 3) when the group tried to figure out how to prove every convergent sequence is a Cauchy sequence, Andy explained "you just have to show that there exists an N. So if you just choose one further down the line, it would still work." If Andy had not yet grasped the dependence of $N$ on $\varepsilon$ in the $\varepsilon-N$ definition, Andy would not be able to think about choosing a different value for $N$ in proving the statement. In addition to Andy's utterance, Dave's gesture of moving his hand along the $x$-axis further to the right, and Ryan's immediate affirmation indicate that the dependence of $N$ on $\varepsilon$ was no longer challenged. We thus regard as valid the inference that the students in the group 
developed collective intuitive understanding of the dependence relationship of $N$ on $\varepsilon$ in the $\varepsilon-N$ definition throughout the IRA course.

\section{Practical Implications to IRA Education}

The present study adds a number of new insights into the teaching and learning of the convergence of a sequence in IRA classrooms. First of all, the $\varepsilon$-strip activity in this study was implemented as an instructional tool in an undergraduate real analysis classroom. Such a classroom environment created a distinct research context from the previous studies as they were mainly clinical interviews or a one-on-one teaching experiments. In fact, the results from these previous studies cannot be applied to explain how students' learning occur via the $\varepsilon$-strip activity in a regular classroom: More complicated group dynamics and interactions among students are involved and students are influenced by, and are influencing, other students' understanding. In particular, in our classroom teaching experiment, the classroom environment with small group discussion for inquiry learning created a distinct research context from the previous studies with individual students. In fact, the interaction among students in our teaching experiment was a catalyst in the development of student understanding which would not be often observed in clinical interviews.

In the classroom reported in this paper, one teacher-researcher had full responsibility of communicating with the entire class unlike previous studies. In addition, the set topics of the course should be covered by the semester-long IRA course. Hence, there was implicit time constraint for the $\varepsilon$-strip activity in the classroom. Unlike the previous studies mentioned above, in our classroom teaching experiment, the $\varepsilon$-strip activity was implemented in one typical class period. The students in the classroom spent 40 minutes to evaluate $\varepsilon$-strip definitions A and B (Episode 1), 20 minutes to compare the $\varepsilon$-strip definitions with the $\varepsilon-N$ definition (Episode 2), and 20 minutes to prove a statement about convergent sequences (Episode 3). It is remarkable that many students in the classroom conceptualized the $\varepsilon-N$ definition for such a short span of time.

The present study also provides empirical data that enhance our understanding of students' conceptualization of the convergence of a sequence. The main focus of this study was on the development of students' intuitive understanding. This point is another difference from the previous studies which paid attention to classification of student conceptions. On the other hand, the present study selected participants from students who had not yet received any formal instruction for the $\varepsilon-N$ definition in order to minimize instructional influence of the $\varepsilon-N$ definition at the initial stage of the development. This contrasts with some of the previous studies selected their participants from those who had already learned the $\varepsilon-N$ definition. A significance of the present study is that it showed the processes of the development of students' intuition to be compatible with the $\varepsilon-N$ definition in a regular classroom. Therefore, we suggest the $\varepsilon$-strip activity as an instructional intervention, proven beneficial in this study, to help students develop their secondary intuitive understanding compatible with the $\varepsilon-N$ definition in an actual IRA classroom environment. 
Acknowledgments This paper is based upon work supported by the National Science Foundation under Grant No. DUEEHR-0837443. Any opinions, findings, and conclusions or recommendations expressed in this paper are those of the authors and do not necessarily reflect the views of the National Science Foundation.

\section{References}

Alcock, L. (2010). Interactions between teaching and research: Developing pedagogical content knowledge for real analysis. In R. Leikin \& R. Zazkis (Eds.), Learning through teaching mathematics (pp. 227-245). Netherlands: Springer.

Alcock, L., \& Weber, K. (2005). Proof validation in real analysis: inferring and checking warrants. Journal of Mathematical Behavior, 24, 125-134.

Barne, J. (2007). Teaching real analysis in the land of make believe. Problems, Resources, and Issues in Mathematics Undergraduate Studies, 17, 366-372.

Bruner, J. (1960). The process of education. Cambridge: Harvard University Press.

Burton, L. (1999). Why is intuition so important to mathematicians but missing from mathematics education? For the Learning of Mathematics, 19, 27-32.

Cheng, K., \& Leung, A. (2015). A dynamic applet for the exploration of the concept of the limit of a sequence. International Journal for Mathematical Education in Science and Technology, 46, 187-204.

Cobb, P., Stephan, M., McClain, K., \& Gravemeijer, K. (2001). Participating in classroom mathematical practices. The Journal of the Learning Sciences, 10, 113-163.

Cornu, B. (1991). Limits. In D. Tall (Ed.), Advanced mathematical thinking (pp. 153-166). Dordrecht: Kluwer.

Cory, B., \& Garofalo, J. (2011). Using dynamic sketches to enhance preservice secondary mathematics teachers' understanding of limits of sequences. Journal for Research in Mathematics Education, 42, $65-96$.

Courant, R., \& Robbins, H. (1963). What is mathematics? An elementary approach to ideas and methods. Oxford: Oxford University Press.

Davis, R., \& Vinner, S. (1986). The notion of limit: some seemingly unavoidable misconception stages. Journal of Mathematical Behavior, 5, 281-303.

Dawkins, P. (2012). Metaphor as a possible pathway to more formal understanding of the definition of sequence convergence. Journal of Mathematical Behavior, 31, 331-343.

Dawkins, P., \& Roh, K. (2016). Promoting metalinguistic and metamathematical reasoning in proof-oriented mathematics courses: a method and a framework. International Journal of Research on Undergraduate Mathematics Education, 2, 197-222.

Durand-Guerrier, V., \& Arsak, G. (2005). An epistemological and didactic study of a specific calculus reasoning rule. Educational Studies in Mathematics, 60, 149-172.

Edwards, B. (1997). Undergraduate mathematics majors' understanding and use of formal definitions in real analysis. Unpublished doctoral dissertation. Pennsylvania State University.

Fischbein, E. (1987). Intuition in science and mathematics. Dordrecht: Reidel.

Fischbein, E. (1994). The interaction between the formal, the algorithmic, and the intuitive comonents in a mathematical activity. In R. Biehler, R. W. Scholz, R. Stässer, \& B. Winkelmann (Eds.), Didactivs of mathematics as a scientific discipline (pp. 231-245). Dordrecht: Kluwer.

Fischbein, E. (1999). Intuition and schemata in mathematical reasoning. Educational Studies in Mathematics, $38,11-50$.

Hadamard, J. (1996). The psychology of invention in the mathematical field. Princeton: Princeton University Press.

Harel, G. (2008). DNR perspective on mathematics curriculum and instruction, Part I: focus on proving. ZDM, 40, 487-500.

Jorgensen, T., \& Shipman, B. (2012). Limits of infinite procesess for liberal arts majors: two classic examples. Problems, Resources, and Issues in Mathematics Undergraduate Studies, 22, 482-499.

Knapp, J. (2006). Students' appropriation of proving practices in advanced calculus. Unpublished doctoral dissertation. Arizona State University.

Knapp, J., \& Roh, K. (2008). Students' notion of convergence in advanced calculus courses. Proceedings of the 11th Conference of the SIGMAA on Research in Undergraduate Mathematics Education. Retrieved from http://sigmaa.maa.org/rume/crume2008, San Diego, CA. 
Mamona-Downs, J. (2001). Letting the intuitive bear on the formal: a didactical approach for the understanidng of the limit of a sequence. Educational Studies in Mathematics, 48, 259-288.

Martin, L., Towers, J., \& Pirie, S. (2006). Collective mathematical understanding as improvisation. Mathematical Thinking and Learning, 8, 149-183.

Navarro, M., \& Carreras, P. (2006). Constructing a concept image of convergence of sequence in the van Hiele framework. Research in Collegiate Mathematics Education, 6, 61-98.

Oehrtman, M., Swinyard, C., \& Martin, J. (2014). Problems and solutions in students' reinvention of a definition for sequence convergence. Journal of Mathematical Behavior, 33, 131-148.

Pinto, M., \& Tall, D. (2002). Building formal mathematics on visual imagery: a case study and a theory. For the Learning of Mathematics, 22, 2-10.

Pollatsek, H., Barker, W., Bressoud, D., Epp, S., Ganter, S., \& Haver, B. (2004). Undergraduate programs and courses in the mathematical sciences: CUPM curriculum guide 2004. Washington, D. C.: Mathematical Association of America.

Przenioslo, M. (2004). Images of the limit of function formed in the course of mathematical studies at the university. Educational Studies in Mathematics, 55, 103-132.

Rasmussen, C., \& Stephan, M. (2008). A methodology for documenting collective activity. In A. Kelly, R. Lesh, \& J. Baek (Eds.), Handbook of design research methods in education: Innovations in science, technology, engineering, and mathematics learning and teaching (pp. 195-215). NY: Routledge.

Rasmussen, C., Stephan, M., \& Allen, K. (2004). Classroom mathematical practices and gesturing. Journal of Mathematical Behavior, 23, 301-323.

Raynolds, F., \& Reeve, R. (2002). Gesture in collaborative mathematical problem-solving. Journal of Mathematical Behavior, 20, 447-460.

Roh, K. (2008a). Students' images and their understanding of definitions of the limit of a sequence. Educational Studies in Mathematics, 69, 217-233.

Roh, K. (2008b). How to help students conceptualize the rigorous definition of the limit of a sequence. Problems, Resources, and Issues in Mathematics Undergraduate Studies, 20, 473-487.

Roh, K. (2010). An empirical study of students' understanding of a logical structure in the definition of limit via the $\varepsilon$-strip activity. Educational Studies in Mathematics, 73, 263-279.

Roh, K., \& Lee, Y. (2011). The Mayan activity: a way of teaching multiple quantifications in logical contexts. Problems, Resources, and Issues in Mathematics Undergraduate Studies, 21, 685-698.

Shipman, B. (2012). A comparative study of definitions on limits and continuity of functions. Problems, Resources, and Issues in Mathematics Undergraduate Studies, 22, 609-633.

Steffe, L., \& Thompson, P. (2000). Teaching experiment methodology: Underlying principles and essential elements. In A. Kelly \& R. Lesh (Eds.), Handbook of research design in mathematics and science education (pp. 267-306). Mahwah: Lawrence Erlbaum Associates.

Stewart, J. (2003). Calculus: Early transcendental single variable (5th ed.). Belmont: Brooks/Cole-Thmas Learning.

Strass, A., \& Corbin, J. (1998). Basics of qualitative research: Techniques and procedures for developing grounded theory (2nd ed.). Thousand Oaks: Sage.

Swinyard, C. (2011). Reinventing the formal definition of limit: the case of Amy and Mike. Journal of Mathematical Behavior, 30, 93-114.

Szydlik, J. E. (2000). Mathematical beliefs and conceptual understanding of the limit of function. Journal for Research in Mathematics Education, 31, 256-276.

Tabach, T., Hershkowitz, R., Rasmussen, C., \& Dreyfus, T. (2014). Knowledge shifts and knowledge agents in the classroom. Journal of Mathematical Behavior, 33, 192-208.

Vidakovic, D., \& Martin, W. (2004). Small-group searches for mathematical proofs and individual reconstructions of mathematical concepts. Journal of Mathematical Behavior, 23, 465-492.

Weber, K. (2004). Traditional instruction in advanced mathematics courses: a case of one professor's lectures and proofs in an introductory real analysis course. Journal of Mathematical Behavior, 23, 115-133.

Yackel, E., \& Cobb, P. (1996). Sociomathematical norms, argumentation, and autonomy in mathematics. Journal for Research in Mathematics Education, 27, 458-477. 